\title{
Aharonov-Bohm Radiation of Fermions
}

\author{
Yi-Zen $\mathrm{Chu}^{1}$, Harsh Mathur ${ }^{1}$ and Tanmay Vachaspati ${ }^{1,2}$ \\ ${ }^{1}$ CERCA, Department of Physics, Case Western Reserve University, \\ 10900 Euclid Avenue, Cleveland, OH 44106-7079, \\ ${ }^{2}$ Institute for Advanced Study, Princeton, NJ 08540
}

\begin{abstract}
We analyze Aharonov-Bohm radiation of charged fermions from oscillating solenoids and cosmic strings. We find that the angular pattern of the radiation has features that differ significantly from that for bosons. For example, fermionic radiation in the lowest harmonic is approximately isotropically distributed around an oscillating solenoid, whereas for bosons the radiation is dipolar. We also investigate the spin polarization of the emitted fermion-antifermion pair. Fermionic radiation from kinks and cusps on cosmic strings is shown to depend linearly on the ultraviolet cut-off, suggesting strong emission at an energy scale comparable to the string energy scale.
\end{abstract}

\section{INTRODUCTION}

The Aharonov-Bohm (AB) interaction [1, 2] between charged particles and thin magnetic fluxes is of much interest as it provides a physical consequence of a pure gauge field with vanishing field strength but non-trivial topology. Further, the physical effects emerge only in quantum theory and hence the $\mathrm{AB}$ interaction provides an example of a quantum, topological interaction.

The classic Aharonov-Bohm setup involves scattering an electron off a solenoid, with non-trivial scattering obtained even for an arbitrarily thin solenoid, whereby the electron is exclusively localized in a region of vanishing magnetic field. A novel feature of the scattering is a periodic dependence of the scattering cross-section on the magnetic flux through the solenoid. If $\Phi$ denotes the magnetic flux in the solenoid and $e$ the electron charge, the cross-section is proportional to $\sin ^{2}(\pi \epsilon)$ where $\epsilon \equiv e \Phi / 2 \pi$.

The classic AB setup was recently extended in another direction [3], where it was shown that an oscillating solenoid in vacuum can produce charged particleantiparticle bosons from the vacuum due to the $\mathrm{AB}$ interaction. The $\mathrm{AB}$ radiation rate also has the characteristic $\sin ^{2}(\pi \epsilon)$ dependence on the magnetic flux.

$\mathrm{AB}$ radiation is relevant to the evolution of cosmic strings, which are similar to solenoids, except the magnetic flux within them is massive, unlike electromagnetic fluxes in laboratory solenoids. Moreover, the gravitational analog of the $\mathrm{AB}$ effect can cause cosmic strings to emit light, even if the fields composing the cosmic string are unrelated to electromagnetic fields [3, 4].

In this paper we will investigate fermionic $\mathrm{AB}$ radiation. One motivation is that the electron is a fermion. Hence fermionic AB radiation is what is relevant to oscillating solenoids. The investigation is also relevant, for instance, to neutrino emission from cosmic strings by the $\mathrm{AB}$ process. A second motivation is that the spin of the fermion adds another degree of freedom to the emission and the polarization properties of the radiation are of interest.

Our results show a significant difference between $\mathrm{AB}$ radiation of bosons and fermions. For example, if a solenoid aligned with the $z$-axis oscillates along the $x$ direction, the angular distribution of bosonic $\mathrm{AB}$ radiation is peaked in the $y$-direction. Fermionic AB radiation, however, is (approximately) isotropically distributed.

The outline of the paper is as follows. In Secs. II through IV we use conventional interaction picture perturbation theory to calculate the fermion-antifermion pair production rate in the small $\mathrm{AB}$ phase $(\epsilon)$ limit. We consider $\mathrm{AB}$ radiation from an infinite straight solenoid oscillating perpendicular to its length in Sec. III, a cosmic string loop with kinks in Sec. IVA and a cosmic string loop with cusps in Sec. IVB In Sec. V we solve the problem using a different technique that does not assume that $\epsilon$ is small. We call this the "moving frames" scheme and use it to obtain the $\sin (\pi \epsilon)$ dependence of the radiation on the $\mathrm{AB}$ phase, provided the motion of the solenoid is slow. We conclude in Sec.VI and describe our conventions in Appendix $\mathrm{A}$.

\section{AB FERMION PAIR PRODUCTION}

\section{A. Setup}

The interaction of fermions with the gauge potential of the thin solenoid or string is contained within the Dirac action

$$
S_{\psi} \equiv \int d^{4} x \bar{\psi}(i \not D-m) \psi
$$

(See Appendix @ for conventions.) The relevant interaction term is

$$
L_{\mathrm{int}}=e A_{\mu} \bar{\psi} \gamma^{\mu} \psi
$$

where $A_{\mu}$ is the classical solution around the flux tube [2]

$$
A_{\nu}=\frac{\Phi}{2} \epsilon_{\mu \nu \alpha \beta} \partial^{\mu} \frac{1}{\partial^{2}} S^{\alpha \beta}
$$

with

$$
\begin{array}{r}
S^{\alpha \beta}(x)=\int d \tau d \sigma \sqrt{-\gamma} \epsilon^{a b} \partial_{a} X^{\alpha} \partial_{b} X^{\beta} \delta^{(4)}(x-X(\sigma, \tau)) \\
=\int d \tau d \sigma\left(\dot{X}^{\alpha} X^{\beta^{\prime}}-\dot{X}^{\beta} X^{\alpha \prime}\right) \delta^{(4)}(x-X(\sigma, \tau))
\end{array}
$$




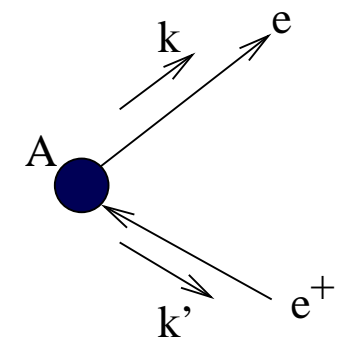

FIG. 1: Feynman diagram for fermion-antifermion (which we have named $e^{+} e^{-}$for convenience) pair production from a magnetic flux tube in motion. The black dot represents the classical gauge field of the flux tube and the solid lines are the outgoing electron and positron states.

and $X^{\mu}(\sigma, \tau)$ gives the position of the flux tube in terms of world-sheet coordinates $\sigma$ and $\tau$.

We will need the Fourier transform of $A_{\mu}$ and this is given by

$$
\tilde{A}_{\nu}=-i \frac{\Phi}{2} \epsilon_{\mu \nu \alpha \beta} \frac{k^{\mu}}{k^{2}} \tilde{S}^{\alpha \beta}
$$

with

$$
\begin{aligned}
\tilde{S}^{\alpha \beta}(k) & =\int d^{4} x e^{+i k \cdot x} S^{\alpha \beta}(x) \\
& =\frac{1}{2} \int d t \int d \sigma \frac{\partial X^{[\alpha}}{\partial t} \frac{\partial X^{\beta]}}{\partial \sigma} e^{i k_{\mu} X^{\mu}}
\end{aligned}
$$

where $\tau=t$ and superscripts within square brackets are antisymmetrized.

In the case of a straight solenoid, we will impose the dynamics by hand and consider oscillatory motion. In the case of a cosmic string, the dynamics will be given by the Nambu-Goto action as discussed in Sec. IV

\section{B. Pair production amplitude}

The amplitude for pair production is then given by the Feynman diagram in Fig. 1.

$$
\begin{aligned}
& \mathcal{M}\left(0 \rightarrow e^{+} e^{-}\right)=\frac{e \Phi}{p^{2}} \epsilon_{\mu \nu \alpha \beta} p^{\mu} \mathcal{J}^{\nu} \widetilde{\mathcal{S}}^{\alpha \beta} \\
& \mathcal{J}^{\nu}\left(k, s ; k^{\prime}, s^{\prime}\right) \equiv \frac{\bar{u}_{k}^{s} \gamma^{\nu} v_{k^{\prime}}^{s^{\prime}}}{\sqrt{2 k_{0}} \sqrt{2 k_{0}^{\prime}}}, \quad p^{\mu}=k^{\mu}+k^{\prime \mu}
\end{aligned}
$$

where the $s$ and $k$ are the spin and momentum labels for the outgoing fermion state and the primed labels are for the anti-fermion.

It will turn out, for all three cases of interest in this paper, $\widetilde{\mathcal{S}}^{\alpha \beta}$ can be factorized into an antisymmetrized product of two independent integrals,

$$
\widetilde{\mathcal{S}}^{\alpha \beta}=\frac{1}{2} I_{+}^{[\alpha} I_{-}^{\beta]}
$$

Furthermore, as can be checked explicitly, the $I_{ \pm}^{\mu}$ and electromagnetic current $\mathcal{J}^{\mu}$ are conserved ${ }^{1}$

$$
p_{\mu} I_{ \pm}^{\mu}=p_{\mu} \mathcal{J}^{\mu}=0
$$

This allows us to re-write

$$
\epsilon_{\mu \nu \alpha \beta} p^{\mu} \mathcal{J}^{\nu} \widetilde{\mathcal{S}}^{\alpha \beta}=\frac{p^{2}}{p_{0}} \mathbf{J} \cdot\left(\mathbf{I}_{+} \times \mathbf{I}_{-}\right)
$$

so that the amplitude now reads

$$
\mathcal{M}=\frac{2 \pi \epsilon}{p_{0}} \mathbf{J} \cdot\left(\mathbf{I}_{+} \times \mathbf{I}_{-}\right), \quad \epsilon \equiv \frac{e \Phi}{2 \pi}
$$

Total rate: Our normalization of the Dirac spinors (A3) is such that the square of the amplitude itself is the total number of fermion-antifermion pairs produced. First let us evaluate the total rate, and hence sum over the spins of the final particles. In squaring the amplitude, we may then exploit the spin sums

$$
\sum_{s} u_{k}^{s} \bar{u}_{k}^{s}=\not k+m, \quad \sum_{s^{\prime}} v_{k^{\prime}}^{s^{\prime}} \bar{v}_{k^{\prime}}^{s^{\prime}}=\not k^{\prime}-m,
$$

and the Clifford algebra $\left\{\gamma^{\mu}, \gamma^{\nu}\right\}=2 \eta^{\mu \nu}$ to deduce,

$$
\sum_{s, s^{\prime}} \mathcal{J}^{\mu}\left(\mathcal{J}^{\nu}\right)^{*}=\frac{k^{\prime \mu} k^{\nu}+k^{\prime \nu} k^{\mu}-\left(m^{2}+k \cdot k^{\prime}\right) \eta^{\mu \nu}}{k_{0} k_{0}^{\prime}}
$$

Within the small $\mathrm{AB}$ phase approximation, the result of this spin sum accounts for the entire difference in angular distribution for the outgoing fermion-antifermion pairs from that of the scalar case in [3]. We see that, even at the level of unpolarized rates, the spin of the particles interacting with the vector potential give rise to significant observational signatures.

For comparison, in the bosonic case the corresponding quantity is [3]

$$
\mathcal{J}^{\mu}\left(\mathcal{J}^{\nu}\right)^{*}=\frac{\left(k-k^{\prime}\right)^{\mu}\left(k-k^{\prime}\right)^{\nu}}{k_{0} k_{0}^{\prime}}
$$

The difference in this expression versus the expression for the fermionic case gives rise to different angular distributions for the $\mathrm{AB}$ radiation of bosons and fermions. In particular, $\mathrm{AB}$ bosonic radiation vanishes when $k^{\mu}=k^{\prime \mu}$ whereas fermionic radiation does not.

Now the square of the amplitude, summed over the possible spins of the outgoing particles, reads

$$
\begin{aligned}
\sum_{s, s^{\prime}}|\mathcal{M}|^{2} & =\frac{(2 \pi)^{2} \epsilon^{2}}{p_{0}^{2} k_{0} k_{0}^{\prime}}\left\{\left|\mathbf{I}_{+} \times \mathbf{I}_{-}\right|^{2}\left(m^{2}+k \cdot k^{\prime}\right)\right. \\
& \left.+\left(\left(\mathbf{I}_{+} \times \mathbf{I}_{-}\right) \cdot \mathbf{k}\left(\mathbf{I}_{+} \times \mathbf{I}_{-}\right)^{*} \cdot \mathbf{k}^{\prime}+\text { c.c. }\right)\right\}
\end{aligned}
$$

\footnotetext{
${ }^{1}$ For $I_{ \pm}^{\alpha}$, see Eq. (33), and more explicitly, 16], 37) and 400. The identity for $\mathcal{J}^{\mu}$ follows from the free massive Dirac equations, $\left(\not k^{\prime}+m\right) v_{k^{\prime}}^{s^{\prime}}=0=\bar{u}_{k}^{s}(\not k-m)$.
} 
with the "c.c." representing complex conjugation of the term preceding it.

The $\left|\mathbf{I}_{+} \times \mathbf{I}_{-}\right|^{2}$ will contain an infinite series involving the square of $\delta$-functions, of the form $\left(\delta\left(p_{0}-\ell \Omega\right)\right)^{2}$ where $\Omega$ is the characteristic frequency of oscillation and $\ell$ is the radiation harmonic. This may be interpreted as a single $\delta$-function multiplied by an infinite constant corresponding to the total duration of time divided by $2 \pi$ :

$$
\left(\delta\left(p_{0}-\ell \Omega\right)\right)^{2} \rightarrow \delta\left(p_{0}-\ell \Omega\right) \frac{T}{2 \pi}
$$

where $T$ is the total time duration over which the radiation is calculated. This can be justified formally by using the integral representation of the $\delta$-function and setting the exponential to unity,

$$
\delta(0) \rightarrow \lim _{T \rightarrow \infty} \lim _{\alpha \rightarrow 0} \int_{-T / 2}^{T / 2} \frac{d t}{2 \pi} e^{i \alpha t}=\frac{T}{2 \pi}
$$

The rate of pair production is then given by $\sum_{s, s^{\prime}}|\mathcal{M}|^{2} / T$, integrated over all kinematically possible momenta of the outgoing particles

$$
\begin{aligned}
\frac{d N}{d t}= & \frac{(2 \pi)^{2} \epsilon^{2}}{T} \int \frac{d^{3} k}{(2 \pi)^{3}} \int \frac{d^{3} k^{\prime}}{(2 \pi)^{3}} \frac{1}{p_{0}^{2} k_{0} k_{0}^{\prime}} \\
\times & \left\{\left|\mathbf{I}_{+} \times \mathbf{I}_{-}\right|^{2}\left(m^{2}+k \cdot k^{\prime}\right)\right. \\
& \left.+\left(\left(\mathbf{I}_{+} \times \mathbf{I}_{-}\right) \cdot \mathbf{k}\left(\mathbf{I}_{+} \times \mathbf{I}_{-}\right)^{*} \cdot \mathbf{k}^{\prime}+\text { c.c. }\right)\right\}
\end{aligned}
$$

Spin-dependence: If one wishes to evaluate the spindependence of the scattering it is convenient to work out the amplitudes for the creation of particles and antiparticles with definite helicity. In other words the particle of momentum $k$ is assumed to have its spin aligned or anti-aligned with the momentum $\mathbf{k}$ corresponding to positive or negative helicity. This is accomplished by evaluating the current $\mathcal{J}\left(k, s ; k^{\prime}, s^{\prime}\right)$ taking the spinors $u_{k}^{s}$ and $v_{k^{\prime}}^{s^{\prime}}$ to be of definite helicity (see Appendix A). An example of such a spin-dependent pair production rate is given in Sec. III.

The spin-dependence of pair production has a general feature that can be deduced immediately from the form of the matrix element $\mathcal{M}$. Let us suppose that $\mathbf{I}_{+} \times \mathbf{I}_{-} \propto \hat{\mathbf{m}}$ where $\hat{\mathbf{m}}$ is a unit vector. This assumption will be seen to be valid below for the cases of a straight oscillating string and for degenerate kinky loops discussed below in Secs. III and IVA For this case one can show that

$$
\mathcal{M} \propto \mathbf{J} \cdot \hat{\mathbf{m}}=\xi_{s}^{\dagger} S_{\Sigma, 0} \xi_{s^{\prime}}+\xi_{s}^{\dagger} \mathbf{S}_{\Sigma} \cdot \boldsymbol{\sigma} \xi_{s^{\prime}}
$$

Here

$$
\begin{aligned}
S_{\Sigma, 0} & =2 i \hat{\mathbf{m}} \cdot\left(\hat{\mathbf{n}}^{\prime} \times \hat{\mathbf{n}}\right) \sinh \frac{\zeta}{2} \sinh \frac{\zeta^{\prime}}{2}, \\
\mathbf{S}_{\Sigma} & =2 \cosh \frac{\zeta}{2} \cosh \frac{\zeta^{\prime}}{2} \hat{\mathbf{m}} \\
& +2 \sinh \frac{\zeta}{2} \sinh \frac{\zeta^{\prime}}{2}\left[(\hat{\mathbf{n}} \cdot \hat{\mathbf{m}}) \hat{\mathbf{n}}^{\prime}+\left(\hat{\mathbf{n}}^{\prime} \cdot \hat{\mathbf{m}}\right) \hat{\mathbf{n}}-\left(\hat{\mathbf{n}} \cdot \hat{\mathbf{n}^{\prime}}\right) \hat{\mathbf{m}}\right] .
\end{aligned}
$$

where we have used

$$
J_{-n}(z)=(-1)^{n} J_{n}(z), \quad n \in\{0, \pm 1, \pm 2, \ldots\}
$$

The rapidity is defined via $\cosh \zeta=k^{0} / m, \cosh \zeta^{\prime}=$ $k^{\prime 0} / m$ and $\hat{\mathbf{n}}$ and $\hat{\mathbf{n}}^{\prime}$ are unit vectors along the directions of the momenta $\mathbf{k}$ and $\mathbf{k}^{\prime}$ respectively. Eq. (11) is a simple consequence of the forms of the spinors $u_{k}^{s}$ and $v_{k^{\prime}}^{s^{\prime}}$ given the spin of the particle in its rest frame; $\xi_{s^{\prime}}$ to the spin of the anti-particle in its rest frame. Thus we see that if the particle is measured to be up along the direction $\mathbf{S}_{\Sigma}$ in its rest frame, the anti-particle will definitely be down along the same direction in its rest frame and vice versa. To see this explicitly, if $\xi_{s}^{T}=(1,0)$ then the amplitude is maximized by taking $\xi_{s^{\prime}}^{T}=(1,0)$. However this choice of spinors means that the particle is spin up and the antiparticle is spin down. Hence there is a definite anticorrelation in the spin of the produced particles.

\section{INFINITE, STRAIGHT SOLENOID}

In this section we will consider the pair production due to an infinite, straight solenoid aligned parallel to the $z$-axis, moving in a sinusoidal fashion along the $x$-axis. Hence, with $\sigma=z$,

$$
\begin{aligned}
X^{\alpha}(t, z) & =(t, \xi(t), 0, z) \\
\xi(t) & \equiv \frac{v_{0}}{\Omega} \sin (\Omega t), \quad \Omega>0
\end{aligned}
$$

Putting (13) into (6) we find that the $t$ - and $z$-integrals may be factorized (into $I_{+}$and $I_{-}$respectively). To evaluate $I_{+}$we express the $\cos (\Omega t)$ in its integrand in terms of exponentials, and perform a cylindrical wave expansion via

$$
e^{i \rho \cos \theta}=\sum_{\ell=-\infty}^{+\infty} i^{\ell} J_{\ell}(\rho) e^{i \ell \theta}
$$

The resulting Bessel functions in $I_{+}$can be combined using the recursion relation

$$
\frac{\nu}{z} J_{\nu}(z)=\frac{1}{2}\left(J_{\nu-1}(z)+J_{\nu+1}(z)\right) .
$$

The integral for $I_{-}$simply gives a $\delta$-function. Together,

$$
\begin{aligned}
& I_{+}^{\alpha}=2 \pi \sum_{\ell=-\infty}^{\infty} \delta\left(p_{0}-\ell \Omega\right)(-1)^{\ell} J_{\ell}\left(p_{x} \frac{v_{0}}{\Omega}\right)\left(\delta_{0}^{\alpha}-\delta_{x}^{\alpha} \frac{p_{0}}{p_{x}}\right) \\
& I_{-}^{\beta}=\delta_{3}^{\beta} 2 \pi \delta\left(p_{z}\right)
\end{aligned}
$$

and therefore

$$
\begin{aligned}
\mathbf{I}_{+} \times \mathbf{I}_{-} & =\hat{\mathbf{y}}(2 \pi)^{2} \\
& \times \sum_{\ell=-\infty}^{\infty} \delta\left(p_{z}\right) \delta\left(p_{0}-\ell \Omega\right)(-1)^{\ell} J_{\ell}\left(p_{x} \frac{v_{0}}{\Omega}\right) \frac{p_{0}}{p_{x}}
\end{aligned}
$$
in Appendix A. Here $\xi_{s}$ is a spinor that corresponds to 
Making use of the formal identity

$$
\left(\delta\left(p_{z}\right)\right)^{2} \rightarrow \delta\left(p_{z}\right) \frac{L}{2 \pi} .
$$

where $L$ is the total length of the solenoid we find that the fermion-antifermion pair production rate (10) per unit length of the solenoid in sinusoidal motion is

$$
\dot{N}^{\prime}=\sum_{\ell=1}^{\infty} \int d^{3} k \int d^{3} k^{\prime} \sum_{s, s^{\prime}= \pm} \frac{d^{7} N\left(k s, k^{\prime} s^{\prime}\right)}{d t d^{3} k d^{3} k^{\prime}}
$$

where the differential rate

$$
\frac{d^{7} N\left(k s, k^{\prime} s^{\prime}\right)}{d t d^{3} k d^{3} k^{\prime}}=\frac{\epsilon^{2}}{(2 \pi)^{2}} \frac{\mathcal{N}\left(k s, k^{\prime} s^{\prime}\right)}{4 k_{0} k_{0}^{\prime} p_{x}^{2}} J_{\ell}^{2}\left(p_{x} \frac{v_{0}}{\Omega}\right) \delta\left(p_{z}\right) \delta\left(p^{0}-\ell \Omega\right)
$$

and $s$ and $s^{\prime}$ represent the helicity of the particle and anti-particle respectively. The quantity $\mathcal{N}$ is given by

$$
\begin{aligned}
\mathcal{N}\left(k s, k^{\prime} s^{\prime}\right) & =\left(k_{0} k_{0}^{\prime}+m^{2}-s s^{\prime}|\mathbf{k}|\left|\mathbf{k}^{\prime}\right|\right) \\
& \times\left[1+\frac{s s^{\prime}}{|\mathbf{k}|\left|\mathbf{k}^{\prime}\right|}\left(k_{z} k_{z}^{\prime}+k_{x} k_{x}^{\prime}-k_{y} k_{y}^{\prime}\right)\right] .
\end{aligned}
$$

Note that in the limit that the particle and anti-particle are ultra-relativistic $\mathcal{N}\left(k s, k^{\prime} s^{\prime}\right)$ is negligible for $s=s^{\prime}$; thus pairs are predominantly produced with opposite helicity in this limit. The pair production rate summed over final state polarization is given by the simpler expression

$$
\begin{aligned}
\mathcal{N}\left(k, k^{\prime}\right) & =\sum_{s, s^{\prime}= \pm} \mathcal{N}\left(k s, k^{\prime} s^{\prime}\right) \\
& =4\left(m^{2}+k_{0} k_{0}^{\prime}-k_{z} k_{z}^{\prime}-k_{x} k_{x}^{\prime}+k_{y} k_{y}^{\prime}\right) \\
& \rightarrow 2\left((\ell \Omega)^{2}-\left(k_{x}+k_{x}^{\prime}\right)^{2}-\left(k_{y}-k_{y}^{\prime}\right)^{2}\right)
\end{aligned}
$$

The second line in (21) is the direct consequence of the term in curly brackets in (10). The third line, which is $k_{z}$-independent, has been obtained from the first by imposing $k_{z}=-k_{z}^{\prime}$ and re-writing $k_{0} k_{0}^{\prime}=(1 / 2)(\ell \Omega)^{2}-$ $(1 / 2) k_{0}^{2}-(1 / 2) k_{0}^{\prime 2}$, using the constraints implied by the $\delta$-functions.

Note that when we square the amplitude we obtain a double sum over harmonics. This collapses to a single sum because $\delta\left(p_{0}-\ell \Omega\right) \delta\left(p_{0}-\ell^{\prime} \Omega\right)$ is zero unless $\ell=\ell^{\prime}$, since the requirement that both the $\delta$-function arguments be null cannot otherwise be satisfied. Since the sum of the positive energies $p_{0}=k_{0}+k_{0}^{\prime}$ cannot be zero or negative, we have also removed all the $\ell \leq 0$ terms in the summation in Eq. (18).

Non-relativistic limit: In the non-relativistic limit, $v_{0} \ll 1$, we may utilize

$$
J_{\nu}(z) \approx \frac{1}{\Gamma(\nu+1)}\left(\frac{z}{2}\right)^{\nu}\left(1+\mathcal{O}\left(z^{2}\right)\right), \quad|z| \ll 1
$$

to see that the contribution to the pair production rate at each harmonic begins at $\mathcal{O}\left(v_{0}^{2 \ell}\right)$ plus corrections of
$\mathcal{O}\left(v_{0}^{2 \ell+2}\right)$. (For $\ell \gg 1$, the $J_{\ell}(z)$ becomes exponentially suppressed; see equation (25).) Therefore, provided that $\Omega>2 m$, the first harmonic is the dominant production channel in the non-relativistic limit.

We first, however, begin by performing a consistency check of our calculations based on the $\ell=1$ term. Using the pair production rate in the form (10), and keeping only the leading order term in the series expansion of $J_{1}$ and eliminating the $k_{x} k_{x}^{\prime}$ and $k_{y} k_{y}^{\prime}$ term by integrating over the appropriate angular coordinates,

$$
\begin{aligned}
\operatorname{Eq~(18)~} \rightarrow & \int_{0}^{\infty} d k k \int_{0}^{\infty} d k^{\prime} k^{\prime} \int_{-\infty}^{\infty} d k_{z} \int_{-\infty}^{\infty} d k_{z}^{\prime} \\
& \times \delta\left(k_{z}+k_{z}^{\prime}\right) \delta\left(k_{0}+k_{0}^{\prime}-\Omega\right) \frac{\epsilon^{2}}{k_{0} k_{0}^{\prime}} \frac{v_{0}^{2}}{4 \Omega^{2}} \\
& \times\left(m^{2}+k_{0} k_{0}^{\prime}+k_{z}^{2}\right)
\end{aligned}
$$

This will match (63) from the small flux limit of the moving frames perturbation theory calculation below.

Now, the differential pair production rate is the integrand in (18). Upon integrating $k_{z}$ (or $k_{z}^{\prime}$ ), the third line in (21) indicates that the only $k_{z}=-k_{z}^{\prime}$ dependence in the integrand occurs in the $k_{0} k_{0}^{\prime}$. Enforcing the constraint $k_{0}+k_{0}^{\prime}=\Omega$ by introducing a Lagrange multiplier we see that the integrand, and hence the emission rate, is maximum at $k_{z}=0$. (This is true of the full relativistic emission.) Applying (22) to $\ell=1$ and going to cylindrical coordinates, $\left(k_{x}, k_{y}\right)=k_{\perp}(\cos \theta, \sin \theta)$ and $\left(k_{x}^{\prime}, k_{y}^{\prime}\right)=k_{\perp}^{\prime}\left(\cos \theta^{\prime}, \sin \theta^{\prime}\right)$, together with the second line of (21) then yields

$$
\begin{aligned}
& \frac{d^{5} \dot{N}}{d k_{\perp} d k_{\perp}^{\prime} d \theta d \theta^{\prime} d k_{z}}\left(\ell=1, k_{z}=k_{z}^{\prime}=0\right) \\
& =\left(\frac{v_{0} \epsilon}{4 \pi \Omega}\right)^{2} \frac{k_{\perp} k_{\perp}^{\prime}}{k_{0} k_{0}^{\prime}} \delta\left(k_{0}+k_{0}^{\prime}-\Omega\right) \\
& \quad \times\left(m^{2}+k_{0} k_{0}^{\prime}-k_{\perp} k_{\perp}^{\prime} \cos \left(\theta+\theta^{\prime}\right)\right)
\end{aligned}
$$

with

$$
k_{0}=\sqrt{\mathbf{k}_{\perp}^{2}+m^{2}}, \quad k_{0}^{\prime}=\sqrt{\mathbf{k}_{\perp}^{\prime 2}+m^{2}}
$$

The differential rate is largest in the $x y$-plane and when the outgoing particles' azimuthal angles are supplementary, $\theta+\theta^{\prime}=\pi$. In contrast, boson emission is maximum along $^{2} \theta+\theta^{\prime}=2 \pi$ and dipolar (see Fig. 2).

We may proceed to employ the second line of (21) to obtain the total rate of production of pairs with energy $\Omega$ per unit length of the infinite flux tube

$$
\dot{N}^{\prime}(\ell=1)=\frac{\epsilon^{2}}{2(2 \pi)^{2}}\left(\frac{v_{0}}{2 \Omega}\right)^{2}
$$

\footnotetext{
2 This corrects [3] where it was mistakenly stated that the maximum emission is along $\theta+\theta^{\prime}=\pi$.
} 

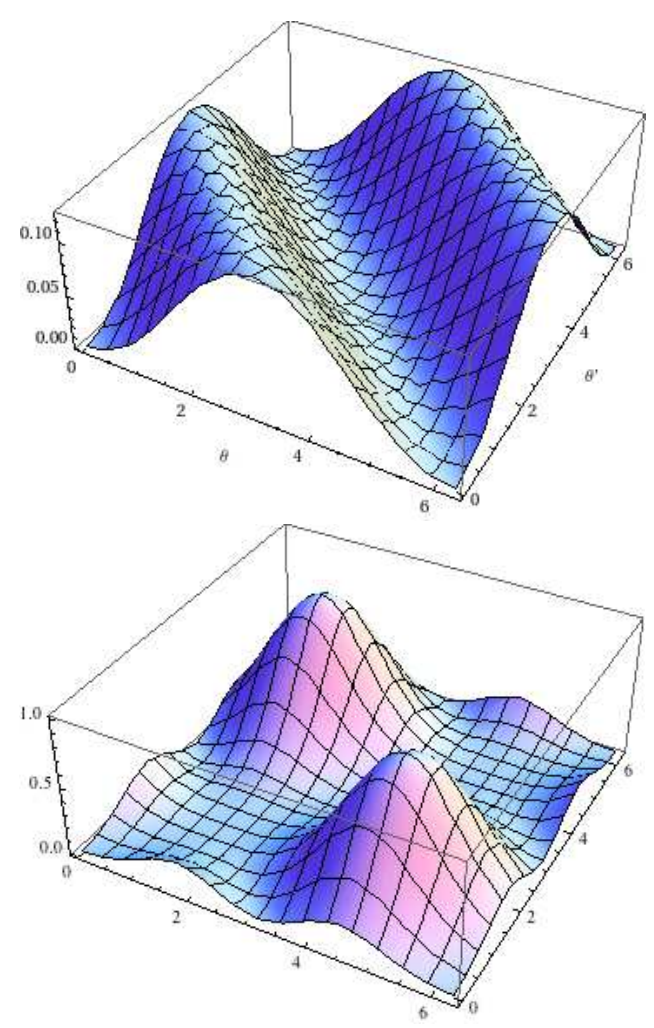

FIG. 2: The radiated power as a function of $\theta$ and $\theta^{\prime}$. The top figure is for fermion radiation $\ell=1$ with $|\boldsymbol{k}|=\ell \Omega / 2=\left|\boldsymbol{k}^{\prime}\right|$, and the lower figure is for bosonic radiation with the same parameters. There is maximum radiation along the line $\theta+\theta^{\prime}=\pi$ in the fermionic case and the radiation is (approximately) circularly symmetric. The bosonic emission is dipolar and along the $\theta+\theta^{\prime}=2 \pi$ line (plot taken from Ref. [3] ).

$$
\begin{aligned}
& \times \int_{m}^{\Omega-m} d k_{0} \int_{-\sqrt{k_{0}^{2}-m^{2}}}^{\sqrt{k_{0}^{2}-m^{2}}} d k_{z} \int_{0}^{2 \pi} d \theta \int_{0}^{2 \pi} d \theta^{\prime} \\
& \times\left[\Omega^{2}-\left(k_{x}+k_{x}^{\prime}\right)^{2}-\left(k_{y}-k_{y}^{\prime}\right)^{2}\right]
\end{aligned}
$$

where

$$
\begin{aligned}
& \left(k_{x}, k_{y}\right)=\sqrt{k_{0}^{2}-m^{2}-k_{z}^{2}}(\cos \theta, \sin \theta) \\
& \left(k_{x}^{\prime}, k_{y}^{\prime}\right)=\sqrt{\left(\Omega-k_{0}\right)^{2}-m^{2}-k_{z}^{2}}\left(\cos \theta^{\prime}, \sin \theta^{\prime}\right)
\end{aligned}
$$

In terms of the mass-to-energy ratio $\tau \equiv 2 \mathrm{~m} / \Omega$, the result is

$$
\begin{gathered}
\dot{N}^{\prime}(\ell=1)=\frac{v_{0}^{2} \epsilon^{2} \Omega^{2}}{384}(2 \sqrt{1-\tau}[16-\tau\{\tau(3 \tau+2)+8\}] \\
\left.-6 \tau^{4} \ln \left[\sqrt{\frac{1}{\tau}-1}+\sqrt{\frac{1}{\tau}}\right]\right)+\mathcal{O}\left(v_{0}^{4}\right)
\end{gathered}
$$

This pair production rate begins at $v_{0}^{2} \epsilon^{2} \Omega^{2} / 12$ for $\tau=0$ and, for increasing $\tau$, decreases monotonically to zero at the threshold $\tau=1$. As the fermion-antifermion pair gets heavier, they become harder to produce.

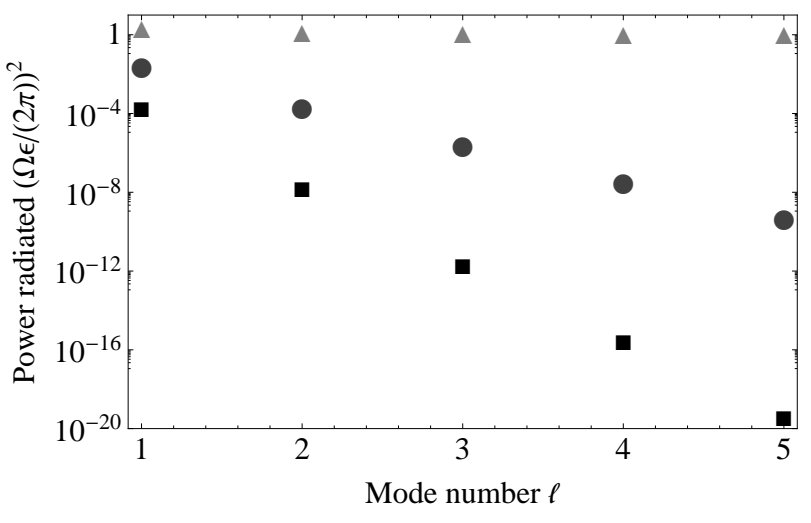

FIG. 3: The radiated power as a function of harmonic for $v_{0}=0.001$ (squares), 0.1 (circles) and 1 (triangles).

At very high harmonics $(\ell \gg 1)$, we may re-scale all momenta by $\ell \Omega / v_{0}$ and denote the new variables by overbars, e.g. $\bar{k} \equiv k v_{0} /(\ell \Omega)$. Then we invoke [5]

$$
J_{\nu}\left(\frac{\nu}{\cosh \alpha}\right) \sim \frac{\exp (-\nu(\alpha-\tanh \alpha))}{\sqrt{2 \pi \nu \tanh \alpha}}, \quad \alpha>0, \quad \nu \gg 1,
$$

to say that, within the integration limits $0<\bar{p}_{x} \lesssim v_{0} \ll$ 1 ,

$$
\left(\frac{J_{\ell}\left(\ell \bar{p}_{x}\right)}{\bar{p}_{x}}\right)^{2} \sim \frac{\exp \left[-2 \ell\left(\alpha_{0}-\tanh \alpha_{0}\right)+2 \ln \left(\cosh \alpha_{0}\right)\right]}{\sqrt{2 \pi \ell \tanh \alpha_{0}}}
$$

where $\alpha_{0}$ is the positive solution to the equation $\cosh \alpha_{0}=1 / \bar{p}_{x}$.

The exponent on the right hand side of the asymptotic expression (26) is a monotonically increasing function of $\bar{p}_{x}$, and takes on large negative values for small $\bar{p}_{x}$ (i.e. large $\alpha_{0}$ ), while tanh $\alpha_{0} \sim 1$. Hence the pair production rate (18) is exponentially small for $\ell \gg 1$, in the nonrelativistic limit. In Fig. 3 we show the power emitted per harmonic for a few values of $v_{0}$.

High frequency limit: When $\Omega \gg 2 m$, such that the fermion-antifermion pairs are produced with very large momentum, to a good approximation, they may be treated as effectively massless. If we re-scale all momenta by the energy, $k \equiv \ell \Omega \bar{k}$ etc., and integrate over $\bar{k}_{\perp}^{\prime}$, the emission rate in the $x y$-plane is

$$
\begin{aligned}
& \frac{d^{4} \dot{N}}{d \bar{k}_{\perp} d \theta d \theta^{\prime} d k_{z}}\left(k_{z}=k_{z}^{\prime}=0, m=0\right) \\
& =\left(\frac{\ell \Omega \epsilon}{2 \pi} \frac{J_{\ell}\left(\ell v_{0} \bar{p}_{x}\right)}{\bar{p}_{x}}\right)^{2} \bar{k}_{\perp}\left(1-\bar{k}_{\perp}\right)\left(1-\cos \left(\theta+\theta^{\prime}\right)\right),
\end{aligned}
$$

with

$$
\bar{p}_{x}=\bar{k}_{\perp} \cos \theta+\left(1-\bar{k}_{\perp}\right) \cos \theta^{\prime}
$$

The rescaled momentum, $\bar{k}_{\perp}$, lies in $(0,1)$ and so $\bar{p}_{x}$ lies in the interval $\left(\cos \theta, \cos \theta^{\prime}\right)$ for $\cos \theta<\cos \theta^{\prime}$, and 
$\left(\cos \theta^{\prime}, \cos \theta\right)$ for $\cos \theta>\cos \theta^{\prime}$. Therefore the absolute value of the argument of $J_{\ell}$ is always less than its order, $\left|\ell v_{0} \bar{p}_{x}\right| \leq \ell v_{0}<\ell$. For $\ell=1$, as a first approximation, we may recall (22), and observe that $\left(J_{\ell}\left(\ell v_{0} \bar{p}_{x}\right) / \bar{p}_{x}\right)^{2}$ stays roughly constant within the range of interest at hand. Hence, the maximum emission occurs for $\bar{k}_{\perp} \approx 1 / 2$ and $\theta+\theta^{\prime}=\pi$. For $\ell \geq 2$, we may employ (15) followed by the fact that both the first turning point and zero of $J_{\ell}(z)$ occurs only for $z \geq \ell$, to argue that $\left(J_{\ell}\left(\ell v_{0} \bar{p}_{x}\right) / \bar{p}_{x}\right)^{2}$ is a monotonically increasing function of $\bar{p}_{x}$ - this means the $\bar{k}_{\perp}$ at which there is maximum emission now shifts away from $1 / 2$, with the direction depending on whether $\cos \theta$ is greater or less than $\cos \theta^{\prime}$.

At higher harmonics, $\ell \gg 1, J_{\ell}$ becomes exponentially suppressed, according to (25) and (26), in most of the $\left(\theta, \theta^{\prime}\right)$ plane. If the motion is relativistic $\left(v_{0} \sim 1\right)$, however, the asymptotic formula [5]

$$
\begin{aligned}
J_{\ell}(z) \sim & \frac{1}{3 \pi}\left(\frac{\sin (\pi / 3) \Gamma(1 / 3)}{(z / 6)^{1 / 3}}\right. \\
& \left.+\frac{\sin (2 \pi / 3) \Gamma(2 / 3)}{(z / 6)^{2 / 3}}(z-\ell)+\ldots\right), \quad \ell \gg 1,
\end{aligned}
$$

valid for $z \sim \ell$, says that $\left(J_{\ell}\left(\ell v_{0} \bar{p}_{x}\right) / \bar{p}_{x}\right)^{2}$ will transition to an inverse power law in $\ell$ and hence peak in the region where $\left|\bar{p}_{x}\right|$ lies closest to unity, namely, where $\cos \theta=$ $\cos \theta^{\prime}= \pm 1$. (Note that $\left(J_{\ell}(-z) /(-z)\right)^{2}=\left(J_{\ell}(z) / z\right)^{2}$.) Therefore, ignoring the $\left(1-\cos \left(\theta+\theta^{\prime}\right)\right)$ factor for now, the peak occurs at $\left(\theta, \theta^{\prime}\right)=(0,0)$ or $(\pi, \pi)$. The shape of the peak is determined by the constant $\bar{p}_{x}$ contour lines on the $\left(\theta, \theta^{\prime}\right)$ plane near $(0,0)$ and $(\pi, \pi)$. They are ellipses with $\bar{k}_{\perp}$-dependent eccentricity, because

$$
\bar{p}_{x}(0,0)=1-\frac{1}{2}\left(\bar{k}_{\perp} \theta^{2}+\left(1-\bar{k}_{\perp}\right) \theta^{\prime 2}\right)+\ldots
$$

$\bar{p}_{x}(\pi, \pi)=-1+\frac{1}{2}\left(\bar{k}_{\perp}(\theta-\pi)^{2}+\left(1-\bar{k}_{\perp}\right)\left(\theta^{\prime}-\pi\right)^{2}\right)+\ldots$

Now we include the effect of the $\left(1-\cos \left(\theta+\theta^{\prime}\right)\right)$ factor which vanishes along the lines $\theta+\theta^{\prime}=2 \pi n$, where $n \in \mathbb{Z}$. The peaks at $(0,0)$ and $(\pi, \pi)$ lie precisely on these lines. So the multiplicative factor $\left(1-\cos \left(\theta+\theta^{\prime}\right)\right)$ modulates the peak and splits it into two, one on either side of the $\theta+\theta^{\prime}=2 \pi n$ lines.

To summarize, at large oscillation frequencies and relativistic solenoid speeds, we expect the high energy spectrum of the fermion-antifermion pairs to be produced predominantly in the $x y$-plane, in narrow pairs of nearly collinear beams, in the $\pm \widehat{x}$ directions. This beaming effect is due to the presence of the Bessel functions $J_{\ell}\left(\ell v_{0} \bar{p}_{x}\right)$, common to both the fermion and boson amplitudes, and hence will occur in the boson case too [3].

\section{COSMIC STRING LOOPS}

We now turn to fermion pair production from cosmic string loops for which the dynamics is given by the
Nambu-Goto action

$$
S_{\mathrm{NG}} \equiv-\mu \int d \tau \int d \sigma \sqrt{-\gamma}
$$

where the world-sheet metric is

$$
\gamma_{a b}=\eta_{\mu \nu} \partial_{a} X^{\mu} \partial_{b} X^{\nu}, \gamma \equiv \operatorname{det}\left(\gamma_{a b}\right)
$$

and $\zeta^{a}=(\tau, \sigma)$ are world-sheet coordinates.

Referring to the Nambu-Goto dynamics for relativistic strings in (28), we will choose $\sigma$ such that constant $t$ - and $\sigma$-lines on the string world sheet are orthogonal, and the energy per unit (proper) length of the string is constant,

$$
X^{\prime} \cdot \dot{X}=0, \quad X^{\prime 2}+\dot{X}^{2}=0 .
$$

These choice of coordinate conditions (30) put the world sheet metric $\gamma_{a b}$ into a conformally flat form, and lead us from the Nambu-Goto action (28) to the wave equation for $X^{\mu}$

$$
\left(\partial_{t}^{2}-\partial_{\sigma}^{2}\right) X^{\mu}(t, \sigma)=0
$$

The $X^{\mu}$ solution may be written as an average of leftand right-movers, $L^{\mu}=L^{\mu}(\sigma+t)$ and $R^{\mu}=R^{\mu}(\sigma-t)$ respectively,

$$
X^{\mu}=\frac{1}{2} L^{\mu}\left(\sigma_{+}\right)+\frac{1}{2} R^{\mu}\left(\sigma_{-}\right)
$$

where we also have introduced world sheet light cone coordinates

$$
\sigma_{ \pm} \equiv \sigma \pm t
$$

and

$$
L^{0}=\sigma_{+}, \quad R^{0}=-\sigma_{-} .
$$

The world sheet coordinate conditions (30) translate into constraints on the spatial components of $X^{\mu}$,

$$
\mathbf{L}^{\prime 2}=\mathbf{R}^{\prime 2}=1
$$

We will focus our attention on two specific loop configurations. The kinky loop has tangent vectors to the string that are discontinuous at isolated points; whereas the cuspy loop has isolated points that reach the speed of light periodically. These configurations satisfy the equations of motion (31) that follow from (28).

If the coordinate length of a given cosmic string loop is $L$, the $\sigma$-integration limits for its corresponding $S^{\mu \nu}$, (see Eq. (6) ) runs from 0 to $L$. Since we have closed loops, $\mathbf{X}$ and derivatives $\partial_{t} X^{\mu}$ and $\partial_{\sigma} X^{\mu}$ are periodic in $\sigma$ as well as $t$. To facilitate the computation, we utilize a formal device introduced in [3] that allows us to factorize $S^{\mu \nu}$ into a product of two one-dimensional integrals, one for each world sheet light cone coordinate. This is based on the observation that, for a periodic function $f$ with period $L$, the integral over one period of $f$ is equivalent 
to the integral over the real line, divided by the (infinite) number of times the former has been over-counted

$$
\int_{0}^{L} d \sigma f(\sigma)=\frac{1}{\delta_{\mathbb{Z}}(0)} \int_{-\infty}^{+\infty} d \sigma f(\sigma),
$$

where

$$
\delta_{\mathbb{Z}}(0) \equiv \frac{\int_{-\infty}^{+\infty} d \sigma e^{i 2 \pi \ell \sigma / L}}{\int_{0}^{L} d \sigma e^{i 2 \pi \ell \sigma / L}}, \quad \ell \in \mathbb{Z}
$$

We may now extend the $\sigma$ integral in $S^{\mu \nu}$ to the entire real line using (32), before changing variables from $(t, \sigma)$ to $\left(\sigma_{+}, \sigma_{-}\right) . S^{\mu \nu}$ then factorizes into

$$
\begin{aligned}
S^{\mu \nu} & =\frac{1}{2} I_{+}^{[\mu} I_{-}^{\nu]} \\
I_{+}^{\alpha} & \equiv \frac{1}{2} \int_{-\infty}^{+\infty} d \sigma_{+} \partial_{+} L^{\alpha} e^{i p \cdot L / 2} \\
I_{-}^{\alpha} & \equiv \frac{1}{2 \delta_{\mathbb{Z}}(0)} \int_{-\infty}^{+\infty} d \sigma_{-} \partial_{-} R^{\alpha} e^{i p \cdot R / 2},
\end{aligned}
$$

where the derivatives are with respect to $\sigma_{ \pm}{ }^{3}$. Periodicity in $\sigma$ and $t$ implies that the integrands in (33) are periodic in $\sigma_{+}$and $\sigma_{-}$and we can replace them in Eq. (6) with their discrete Fourier series expansions,

$$
\begin{aligned}
& I_{+}^{\alpha}=2 \sum_{\ell=-\infty}^{\infty} \delta\left(p_{0}+\frac{4 \pi}{L} \ell\right) \\
& \times \int_{0}^{L} \frac{d \sigma_{+}}{L} \partial_{+} L^{\alpha} e^{-i \ell 2 \pi \sigma_{+} / L} e^{-i \mathbf{p} \cdot \mathbf{L} / 2} \\
& I_{-}^{\alpha}=\frac{2 \pi}{\delta_{\mathbb{Z}}(0)} \sum_{\ell=-\infty}^{\infty} \delta\left(p_{0}-\frac{4 \pi}{L} \ell\right) \\
& \quad \times \int_{0}^{L} \frac{d \sigma_{-}}{L} \partial_{-} R^{\alpha} e^{-i 2 \pi \ell \sigma_{-} / L} e^{-i \mathbf{p} \cdot \mathbf{R} / 2}
\end{aligned}
$$

The $\mathbf{I}_{+} \times \mathbf{I}_{-}$that follows from (34) will be an infinite sum involving $\left(\delta\left(p_{0}-\ell 4 \pi / L\right)\right)^{2}$. We write

$$
\left(\delta\left(p_{0}-\ell 4 \pi / L\right)\right)^{2}=\delta\left(p_{0}-\ell 4 \pi / L\right) \delta_{\mathbb{Z}}(0) \frac{L}{4 \pi}
$$

and the $\delta_{\mathbb{Z}}(0)$ in (35) will cancel that in $I_{-}$(34).

\footnotetext{
${ }^{3}$ If we had not applied (32), upon converting to light cone coordinates, the inner $\sigma_{ \pm}$-integration would have limits that depend on the outer integration variable, and $S^{\mu \nu}$ would not factorize.
}

\section{A. Kinky Loops}

The "degenerate" kinky loop solution we will consider is

$$
\begin{array}{r}
\mathbf{L}\left(\sigma_{+}\right)= \begin{cases}\sigma_{+} \mathbf{A} & 0 \leq \sigma_{+} \leq \frac{L}{2} \\
\left(L-\sigma_{+}\right) \mathbf{A} & \frac{L}{2} \leq \sigma_{+} \leq L\end{cases} \\
\mathbf{R}\left(\sigma_{-}\right)= \begin{cases}\sigma_{-} \mathbf{B} & 0 \leq \sigma_{-} \leq \frac{L}{2} \\
\left(L-\sigma_{-}\right) \mathbf{B} & \frac{L}{2} \leq \sigma_{-} \leq L\end{cases}
\end{array}
$$

where $\mathbf{A}$ and $\mathbf{B}$ are unit vectors. This loop is degenerate because it consists of four straight segments and is kinky because of its four corners. The four straight segments propagate with constant speed but shrink and expand due to the motion of the kinks.

Now, denoting

$$
p_{\mathrm{A}} \equiv p_{i} \mathbf{A}^{i}, \quad p_{\mathrm{B}} \equiv p_{i} \mathbf{B}^{i},
$$

and putting the kinky loop trajectory (36) into (34) then leads to

$$
\begin{aligned}
I_{+}^{\alpha}= & \sum_{\ell=-\infty}^{\infty}\left(p_{\mathrm{A}},-p_{0} \mathbf{A}\right) \delta\left(p_{0}-\frac{4 \pi}{L} \ell\right) \\
& \times \frac{16 \pi e^{i\left(p_{\mathrm{A}} L / 8-\pi \ell / 2\right)}}{L\left(p_{\mathrm{A}}^{2}-p_{0}^{2}\right)} \sin \left(\frac{p_{\mathrm{A}}}{8} L-\frac{\pi}{2} \ell\right) \\
I_{-}^{\alpha}= & \frac{1}{\delta_{\mathbb{Z}}(0)} \sum_{\ell=-\infty}^{\infty}\left(-p_{\mathrm{B}}, p_{0} \mathbf{B}\right) \delta\left(p_{0}-\frac{4 \pi}{L} \ell\right) \\
& \times \frac{16 \pi e^{i\left(p_{\mathrm{B}} L / 8+\pi \ell / 2\right)}}{L\left(p_{\mathrm{B}}^{2}-p_{0}^{2}\right)} \sin \left(\frac{p_{\mathrm{B}}}{8} L+\frac{\pi}{2} \ell\right)
\end{aligned}
$$

and

$$
\begin{aligned}
\mathbf{I}_{+} \times & \mathbf{I}_{-}=-16 \sum_{\ell=-\infty}^{\infty} p_{0}^{2} \frac{4 \pi}{L} \frac{e^{i\left(p_{\mathrm{A}}+p_{\mathrm{B}}\right) L / 8}}{\left(p_{\mathrm{A}}^{2}-p_{0}^{2}\right)\left(p_{\mathrm{B}}^{2}-p_{0}^{2}\right)} \\
& \times \sin \left(\frac{p_{\mathrm{A}}}{8} L-\frac{\pi}{2} \ell\right) \sin \left(\frac{p_{\mathrm{B}}}{8} L+\frac{\pi}{2} \ell\right) \\
& \times \delta\left(p_{0}-\frac{4 \pi}{L} \ell\right) \mathbf{A} \times \mathbf{B}
\end{aligned}
$$

The power radiated from the kinky loop due to fermion pairs emitted with energy $\ell \Omega$, for a fixed $\ell$, is the corresponding pair production rate multiplied by the energy $4 \pi \ell / L$

$$
\begin{aligned}
& \dot{E}_{\ell}^{(\mathrm{K})}=2 \pi \epsilon^{2}\left(\frac{64 \pi}{L}\right)^{2}\left(\frac{4 \pi \ell}{L}\right)^{3} \int \frac{d^{3} k}{(2 \pi)^{3}} \frac{d^{3} k^{\prime}}{(2 \pi)^{3}} \frac{1}{k_{0} k_{0}^{\prime}} \\
& \times \frac{\sin ^{2}\left(p_{\mathrm{A}} L / 8-\pi \ell / 2\right) \sin ^{2}\left(p_{\mathrm{B}} L / 8+\pi \ell / 2\right)}{\left(p_{\mathrm{A}}^{2}-p_{0}^{2}\right)^{2}\left(p_{\mathrm{B}}^{2}-p_{0}^{2}\right)^{2}} \\
& \times\left[(\mathbf{A} \times \mathbf{B})^{2}\left(m^{2}+k \cdot k^{\prime}\right)+2 \mathbf{A} \times \mathbf{B} \cdot \mathbf{k} \mathbf{A} \times \mathbf{B} \cdot \mathbf{k}^{\prime}\right] \\
& \times \delta\left(p_{0}-\frac{4 \pi}{L} \ell\right)
\end{aligned}
$$

The total power radiated is then

$$
\dot{E}^{(\mathrm{K})}(N)=\sum_{\ell=1}^{N} \dot{E}_{\ell}^{(\mathrm{K})}
$$




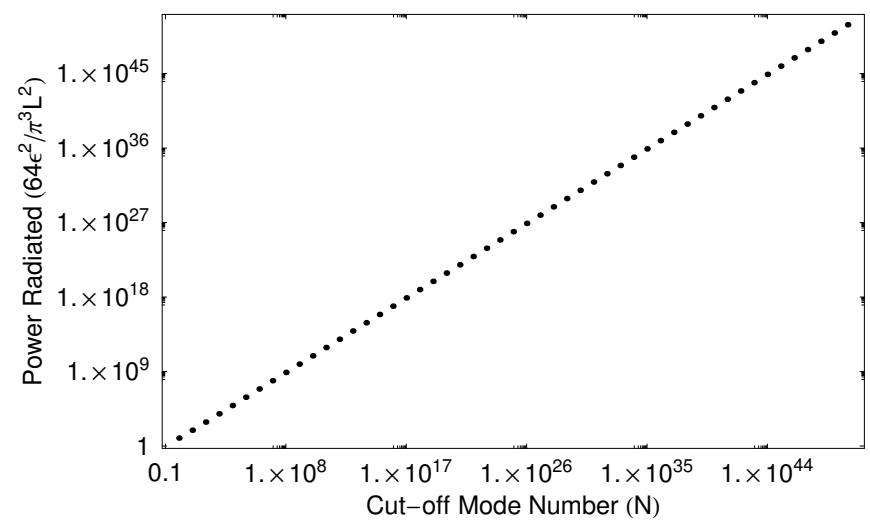

FIG. 4: Plot of power radiated in the form of massless fermion-antifermion pairs in units of $64 \epsilon^{2} /\left(\pi^{3} L^{2}\right)$, from the degenerate kinky loop with $\mathbf{A}$ and $\mathbf{B}$ perpendicular, as a function of the cut-off mode number $N$.

We have truncated the summation at some large integer $N$ because it will turn out, just like in the bosonic case [3], that the total power obtained from summing to $\ell=\infty$ will diverge. The cut-off is related to the rounding off of the kink and may be estimated as the ratio of the length of the cosmic string loop, $L$, to its width $w$. Taking $L \sim 1 \mathrm{Mpc}$ and $w \sim 1 \mathrm{TeV}^{-1}$, we obtain $N \sim 10^{41}$. For the electron - the lightest electrically charged fermion - note that the relevant range of mode number is $10^{35} \lesssim \ell_{e^{+} e^{-}} \lesssim 10^{41}$ for such an $L$ and $w$, where the lower limit is determined by the product of the electron mass and $L$. In the high energy range of the spectrum, which gives the dominant contribution to the power emitted, the fermions can be treated as effectively massless.

When $m=0$, by re-scaling the momenta in the integral (38) via $\left(k, k^{\prime}\right) \equiv(4 \pi \ell / L)\left(\bar{k}, \bar{k}^{\prime}\right)$, the sole dependence on the summation index $\ell$ occurs in the trignometric functions, which in turn can be summed using the formula

$$
\begin{aligned}
& \sum_{\ell=1}^{N} \sin ^{2}(\ell x) \sin ^{2}(\ell y)=\frac{M}{8}-\frac{\sin (M x)}{8 \sin x}-\frac{\sin (M y)}{8 \sin y} \\
& +\frac{\sin (M(x-y))}{16 \sin (x-y)}+\frac{\sin (M(x+y))}{16 \sin (x+y)}, \quad M \equiv 2 N+1
\end{aligned}
$$

The resulting integral was then evaluated numerically with Mathematica.

In Fig. 4 we have plotted the total power $\dot{E}(N)$ as a function of the cut-off $N$, for $m=0$ and for the "square" loop which has $\mathbf{A} \cdot \mathbf{B}=0$, from $N=1$ up to $N=10^{50}$. We see that the power radiated per mode $\ell$ is indeed independent of $\ell$, in this massless limit, and hence the total power emitted grows linearly with $N \sim L / w$ and can be very large.

\section{B. Cuspy Loops}

The cuspy loop solution we will consider is

$$
\begin{aligned}
\mathbf{L}\left(\sigma_{+}\right) & =\frac{L}{2 \pi}\left(\sin \left(\frac{2 \pi \sigma_{+}}{L}\right), 0,-\cos \left(\frac{2 \pi \sigma_{+}}{L}\right)\right) \\
\mathbf{R}\left(\sigma_{-}\right) & =\frac{L}{2 \pi}\left(\sin \left(\frac{2 \pi \sigma_{-}}{L}\right),-\cos \left(\frac{2 \pi \sigma_{-}}{L}\right), 0\right)
\end{aligned}
$$

The loop has cusps because there are points such that $\partial_{+} \mathbf{L}=-\partial_{-} \mathbf{R}$. These occur at $2 \pi \sigma_{ \pm} / L=0, \pi$ at which point the velocity of the string is $\pm \hat{\mathbf{x}}$, i.e. the point on the string reaches the speed of light.

Putting the cuspy loop trajectory (39) into (34), combining the sines and cosines occurring in the exponential into one trigonometric function before performing a cylindrical wave expansion with (14) then gives us

$$
\begin{aligned}
I_{+}^{\alpha} & =2 \pi \sum_{\ell=-\infty}^{\infty}\left(\varphi_{(+\mid-\ell)}, i \frac{4 \pi}{L} \hat{\mathbf{y}} \times \vec{\partial}_{p} \varphi_{(+\mid-\ell)}\right) \delta\left(p_{0}-\frac{4 \pi}{L} \ell\right) \\
I_{-}^{\alpha} & =\frac{2 \pi}{\delta_{\mathbb{Z}}[0]} \sum_{\ell=-\infty}^{\infty}\left(\varphi_{(-\mid \ell)}, i \frac{4 \pi}{L} \hat{\mathbf{z}} \times \vec{\partial}_{p} \varphi_{(-\mid \ell)}\right) \delta\left(p_{0}-\frac{4 \pi}{L} \ell\right)
\end{aligned}
$$

with

$$
\begin{aligned}
\varphi_{(+\mid \ell)}\left(p_{x}, p_{z}\right) & =i^{\ell} J_{\ell}\left(\frac{L}{4 \pi} \sqrt{p_{x}^{2}+p_{z}^{2}}\right) \exp \left(i \ell \arctan \left(\frac{p_{x}}{p_{z}}\right)\right) \\
\varphi_{(-\mid \ell)}\left(p_{x}, p_{y}\right) & =-i^{\ell} J_{\ell}\left(\frac{L}{4 \pi} \sqrt{p_{x}^{2}+p_{y}^{2}}\right) \exp \left(i \ell \arctan \left(\frac{p_{x}}{p_{y}}\right)\right) \\
\vec{\partial}_{p} & \equiv\left(\frac{\partial}{\partial p_{x}}, \frac{\partial}{\partial p_{y}}, \frac{\partial}{\partial p_{z}}\right)
\end{aligned}
$$

as well as

$$
\mathbf{I}_{+} \times \mathbf{I}_{-}=-\frac{16 \pi^{3}}{L} \sum_{\ell=-\infty}^{\infty} \delta\left(p_{0}-\frac{4 \pi}{L} \ell\right) \mathbf{n}_{\ell}
$$

with

$$
\mathbf{n}_{\ell} \equiv\left(\hat{\mathbf{y}} \times \vec{\partial}_{p} \varphi_{(+\mid-\ell)}\right) \times\left(\hat{\mathbf{z}} \times \vec{\partial}_{p} \varphi_{(-\mid \ell)}\right)
$$

The derivatives on $J_{\ell}$ occuring in $\mathbf{I}_{ \pm}$and $\mathbf{n}_{\ell}$ may be carried out with the aid of

$$
\partial_{z} J_{\nu}(z)=\frac{1}{2}\left(J_{\nu-1}(z)-J_{\nu+1}(z)\right)
$$

The power radiated from the cuspy loop due to fermion pairs emitted with energy $\ell \Omega$, for a fixed $\ell$, is the corresponding pair production rate multiplied by the energy $4 \pi \ell / L$,

$$
\begin{gathered}
\dot{E}_{\ell}^{(\mathrm{C})}=\int d^{3} k \int d^{3} k^{\prime} \frac{2}{\ell L} \frac{\epsilon^{2}}{k_{0} k_{0}^{\prime}} \delta\left(p_{0}-\frac{4 \pi}{L} \ell\right) \\
\times\left\{\left|\mathbf{n}_{\ell}\right|^{2}\left(m^{2}+k \cdot k^{\prime}\right)+\left(\mathbf{n}_{\ell} \cdot \mathbf{k} \mathbf{n}_{\ell}^{*} \cdot \mathbf{k}^{\prime}+\text { c.c. }\right)\right\}
\end{gathered}
$$




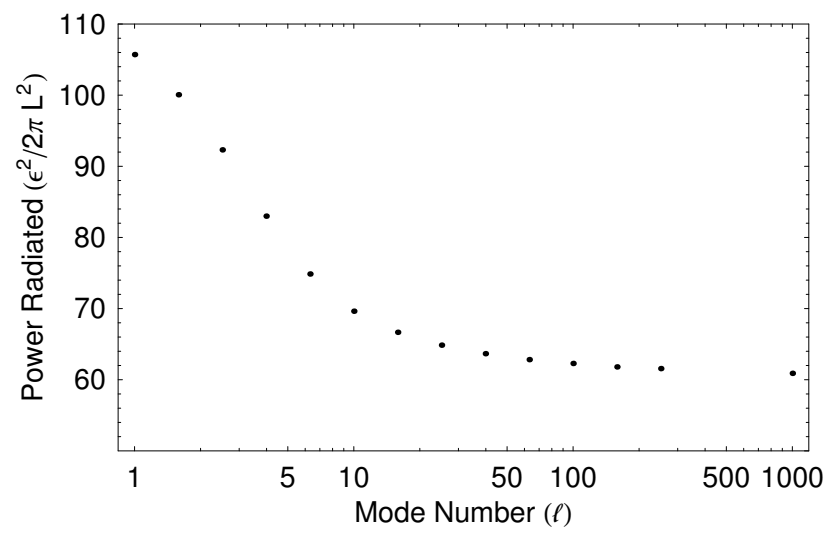

FIG. 5: Plot of power radiated in the form of massless fermion-antifermion pairs in units of $\epsilon^{2} /\left(2 \pi L^{2}\right)$, from the cuspy loop, as a function of the mode number $l$.

Using Mathematica, we evaluated (42) numerically for $\ell=1$ through $\ell=1000$ and $m=0$. Fig. 5 displays the resulting power radiated in the form of massless fermions at each harmonic, with energy $4 \pi \ell / L$. This provides evidence that, at large mode numbers $(\ell \gg 1)$, the power radiated varies very slowly with $\ell$; though a thorough analysis would have to employ more sophisticated numerical techniques (or semi-analytic ones, using (25) and (27)) to evaluate (42) for the astrophysically relevant range of $10^{30} \lesssim \ell \lesssim 10^{50}$.

\section{INFINITE, STRAIGHT SOLENOID VIA MOVING FRAMES PERTURBATION THEORY}

In the second half of this paper, we provide an alternate calculation of the fermion pair production rate from the infinite straight solenoid, introduced in Sec. III, aligned with the $z$-axis, moving in sinusoidal motion along the $x$-axis. The spatial location of the solenoid as a function of time $t$ is given by $\mathbf{X}(t, z)=(\xi(t), 0, z)$, with $\xi(t)=\left(v_{0} / \Omega\right) \sin (\Omega t)$. This non-relativistic computation will only capture the first harmonic of the infinite sum obtained in Sec. III- see (44) below - but will make manifest the periodic dependence of the pair production rate on the AB phase $\epsilon=e \Phi / 2 \pi$ that is expected from such a topological interaction. It will also serve as a consistency check on the results in Sec. III.

Let $\chi_{(s \mid+)}(\mathbf{x}) e^{-i E_{s} t}$ denote the positive and $\chi_{(s \mid-)}(\mathbf{x}) e^{+i E_{s} t}$ the negative energy solutions to the Dirac equation in the presence of the gauge potential $A_{\mu}$ of a static, infinite straight solenoid aligned along the $z$-axis centered at $(x, y)=(0,0)$. (The subscripts $s$ and $s^{\prime}$ in this section denote, collectively, all the rest of labels that come with the solutions.) We will expand the Dirac operator, $\psi$, in terms of "shifted" mode functions, $\chi_{(s \mid \pm)}(x-\xi, y, z) e^{\mp i E_{s} t}$. Then we can show, as derived in Sec. $\mathrm{VA}$ that the fermion-antifermion pair production amplitude for a solenoid moving along the $x$-axis is provided by the product of the integrals

$$
\begin{aligned}
{ }_{\text {out }}\left\langle s, s^{\prime} \mid 0\right\rangle_{\text {in }} & \approx-\int_{-\infty}^{+\infty} d t^{\prime} \dot{\xi}\left(t^{\prime}\right) e^{i\left(E_{s^{\prime}}+E_{s}\right) t^{\prime}} \\
& \times \int d^{3} x^{\prime} \chi_{(s \mid+)}^{\dagger}\left(\mathbf{x}^{\prime}\right) \partial_{x} \chi_{\left(s^{\prime} \mid-\right)}\left(\mathbf{x}^{\prime}\right),
\end{aligned}
$$

where the left-hand side of the equation is an inner product in the Heisenberg picture.

If we specialize to the sinusoidal trajectory considered in Sec. III, the time integral in (43) can be evaluated immediately to yield conservation of energy

$$
\int_{-\infty}^{+\infty} d t^{\prime} \dot{\xi}\left(t^{\prime}\right) e^{i\left(E_{s^{\prime}}+E_{s}\right) t^{\prime}}=-i \pi v_{0} \delta\left(E_{s}+E_{s^{\prime}}-\Omega\right)
$$

(The $\delta\left(E_{s}+E_{s^{\prime}}+\Omega\right)$ term was dropped because, for $\Omega>0$, the sum of the two positive energies $E_{s}$ and $E_{s^{\prime}}$ will never be negative.) The presence of the overall factor $v_{0}$ in (44) and the absence of any dependence on the trajectory in the volume integral in (43) tells us that the perturbative scheme in this section is non-relativistic.

We will proceed to solve for the complete set of modes, $\chi_{(s \mid \pm)}(\mathbf{x}) e^{\mp i E_{s} t}$, evaluating (43) for necessary combinations of $s$ and $s^{\prime}$, before summing the squares of the resulting amplitudes to obtain the pair production rates (62) and (63).

\section{A. Derivation of Equation (43)}

We expand the Dirac operator, within the Heisenberg picture, in terms of $\chi_{(s \mid \pm)}(x-\xi, y, z) e^{\mp i E_{s} t}$

$$
\begin{aligned}
\psi(t, \mathbf{x})=\sum_{s}\left[\alpha_{s}(t) \chi_{(s \mid+)}(x-\xi, y, z) e^{-i E_{s} t}\right. \\
\left.+\beta_{s}^{\dagger}(t) \chi_{(s \mid-)}(x-\xi, y, z) e^{+i E_{s} t}\right]
\end{aligned}
$$

The $\alpha_{s}(t)$ and $\beta_{s}^{\dagger}(t)$ are time-dependent operators that obey appropriate anti-commutation relations and ensure positivity of the Hamiltonian. They also satisfy the equations of motion obtained from the Heisenberg equations of motion:

$$
\begin{aligned}
i \partial_{t} \psi & =h_{\mathrm{D}} \psi \\
h_{\mathrm{D}} & \equiv e A_{0}-i \gamma^{0} \gamma^{j} D_{j}+m \gamma^{0}
\end{aligned}
$$

For a solenoid at rest at $\xi=0$, the operators

$$
a_{s} \equiv \alpha_{s}(t \rightarrow-\infty), \quad b_{s} \equiv \beta_{s}(t \rightarrow-\infty)
$$

acting on the zero particle state at $t \rightarrow-\infty$ destroy, respectively, a fermion $\left(a_{s}\right)$ and an antifermion $\left(b_{s}\right)$ associated with the wavefunctions $\chi_{(s \mid \pm)}(\mathbf{x})$. In particular,

$$
\left\{a_{s}, a_{s^{\prime}}^{\dagger}\right\}=\delta_{s, s^{\prime}}, \quad\left\{b_{s}, b_{s^{\prime}}^{\dagger}\right\}=\delta_{s, s^{\prime}}
$$


Then, to leading order in the Hamiltonian, the zero particle to two particle amplitude is

$$
\begin{aligned}
& \text { out }\left\langle s, s^{\prime} \mid 0\right\rangle_{\text {in }} \\
& \approx-i\left\langle 0 ; t=-\infty\left|a_{s} b_{s^{\prime}} \int d t d^{3} x \psi^{\dagger} i \partial_{t} \psi\right| 0 ; t=-\infty\right\rangle
\end{aligned}
$$

In (46), we have re-expressed the Dirac Hamiltonian density

$$
\mathcal{H}=\psi^{\dagger} h_{\mathrm{D}} \psi=\psi^{\dagger} i \partial_{t} \psi
$$

using its Heisenberg equations of motion (45). (This is legitimate because the $\psi$ 's appearing in the amplitude (46) are operator solutions to the Dirac equation.) The reason for replacing $h_{\mathrm{D}}$ with $i \partial_{t}$ is the following. Since the time evolution of the $\alpha_{s}(t)$ and $\beta_{s}(t)$ away from $a_{s}$ and $b_{s}$ are determined by the interaction between $\psi$ and $A_{\mu}$, to lowest order in interaction, we may now approximate

$$
\alpha_{s}(t) \approx a_{s}, \quad \beta_{s}(t) \approx b_{s}
$$

so that

$$
\begin{aligned}
\left\{a_{s}, \psi^{\dagger}\right\} & =\chi_{(s \mid+)}(x-\xi, y, z) e^{-i E_{s} t} \\
\left\{b_{s}, \psi\right\} & =\chi_{(s \mid-)}(x-\xi, y, z) e^{+i E_{s} t}
\end{aligned}
$$

By anticommuting the $a_{s}$ and $b_{s^{\prime}}$ in (46) to the right, and noting that - because the solenoid is moving solely in the $x$-direction -

$$
\partial_{t} \chi(x-\xi(t), y, z)=-\dot{\xi}(t) \partial_{x} \chi(x-\xi(t), y, z),
$$

we then arrive at (43) after shifting integration variables $x \rightarrow x-\xi$.

\section{B. Modes with $k_{z}=0$}

The solution for the gauge field of an infinite straight solenoid along the $z$-axis with $\sigma=z$ has only two nonzero components

$$
\begin{gathered}
F_{0 y}=E^{y}=\Phi \dot{\xi} \delta(x-\xi(t)) \delta(y), \\
F_{x y}=-B^{z}=-\Phi \delta(x-\xi(t)) \delta(y),
\end{gathered}
$$

The gauge potential that yields (47) is, in Cartesian coordinates,

$$
A_{\mu}=(0,0,-\Phi \Theta(x-\xi(t)) \delta(y), 0)
$$

We wish to solve for the fermionic modes, $\chi$, in the stationary solenoid $(\xi(t)=0)$ case. It is easier to do so using the Lorenz gauge, where in cylindrical coordinates,

$$
A_{\theta}^{(\mathrm{S})}=\frac{\Phi}{2 \pi}, A_{0}^{(\mathrm{S})}=A_{r}^{(\mathrm{S})}=A_{z}^{(\mathrm{S})}=0 .
$$

where the superscript $S$ denotes "stationary".
With the gauge potential determined, the Dirac equation now reads ${ }^{4}$

$$
\left[i \gamma^{\mu}\left(\partial_{\mu}+i e A_{\mu}^{(\mathrm{S})}\right)+m\right] \chi e^{-i E t}=0
$$

Translational symmetry along $z$ implies that $\chi$ has the form

$$
\chi(\mathbf{x})=\varrho(r, \theta) e^{i k_{z} z}
$$

Following Alford and Wilczek 2], to exploit the $z$ translational symmetry, it helps to find a set of $\left\{\gamma^{\mu}\right\}$ matrices such that all of them except $\gamma^{3}$ are block diagonal. This way, in the reference frame where there is no momentum along $z$, i.e. $k_{z}=0$, the Dirac equation splits into a pair of coupled equations, each involving only 2 component spinors. The set of $\left\{\gamma^{\mu}\right\}$ we will use here are defined relative to the ones in the chiral basis $\left\{\gamma_{c}^{\mu}\right\}$ in (A1) as $\gamma^{\mu} \equiv U \gamma_{c}^{\mu} U^{\dagger}$ with

$$
U \equiv \frac{1}{\sqrt{2}}\left[\begin{array}{cccc}
0 & -i & 0 & i \\
-1 & 0 & -1 & 0 \\
0 & -1 & 0 & -1 \\
i & 0 & -i & 0
\end{array}\right]
$$

The new $\gamma^{\mu}$ are

$$
\begin{array}{ll}
\gamma^{0}=\left[\begin{array}{cc}
-\sigma^{3} & 0 \\
0 & \sigma^{3}
\end{array}\right], & \gamma^{1}=\left[\begin{array}{cc}
i \sigma^{1} & 0 \\
0 & -i \sigma^{1}
\end{array}\right] \\
\gamma^{2}=\left[\begin{array}{cc}
-i \sigma^{2} & 0 \\
0 & i \sigma^{2}
\end{array}\right], & \gamma^{3}=\left[\begin{array}{cc}
0 & -i \mathbb{I} \\
-i \mathbb{I} & 0
\end{array}\right]
\end{array}
$$

Now the $k_{z}=0$ solutions are

$$
\chi_{k}(t, r, \theta, z)=\left[\begin{array}{l}
\chi_{A} \\
\chi_{B}
\end{array}\right] e^{-i \omega_{ \pm} t}, \omega_{ \pm} \equiv \pm \sqrt{k^{2}+m^{2}},
$$

with

$$
\begin{aligned}
\chi_{\mathrm{A}}^{\left(\sigma_{1}\right)} & =\mathcal{N}_{\mathrm{A}} e^{i n_{1} \theta}\left[\begin{array}{c}
J_{\sigma_{1}\left(n_{1}+1+\epsilon\right)}(k r) e^{i \theta} \\
\frac{\sigma_{1} k}{m+\omega_{ \pm}} J_{\sigma_{1}\left(n_{1}+\epsilon\right)}(k r)
\end{array}\right], \\
\chi_{\mathrm{B}}^{\left(\sigma_{2}\right)} & =\mathcal{N}_{\mathrm{B}} e^{i n_{2} \theta}\left[\begin{array}{c}
\frac{\sigma_{2} k}{m+\omega_{ \pm}} J_{\sigma_{2}\left(n_{2}+1+\epsilon\right)}(k r) e^{i \theta} \\
J_{\sigma_{2}\left(n_{2}+\epsilon\right)}(k r)
\end{array}\right],
\end{aligned}
$$

where $\sigma_{1,2}=\operatorname{sgn}\left(n_{1,2}+\epsilon\right), n_{1,2} \in \mathbb{Z}, \epsilon=e \Phi / 2 \pi$ and the normalization factors $\mathcal{N}_{\mathrm{A}, \mathrm{B}} \in \mathbb{C}$. The spinors $\chi_{\mathrm{A}}$ and $\chi_{\mathrm{B}}$ are the two independent solutions for each set of positive or negative energy states and we have chosen our $z$-axis so that $\epsilon=e \Phi /(2 \pi)>0$.

For $n_{1,2}+\epsilon>0$ and $n_{1,2}+\epsilon<-1$, the signs $\sigma_{1,2}$ in the indices of the Bessel functions are fixed by the requirement that the solutions $\chi_{k}$ must be square normalizable.

\footnotetext{
${ }^{4}$ In this section, the mass term in the Dirac equation has a + sign, as opposed to the - sign implied by (1). To convert solutions for one into solutions for the other, multiply the Dirac spinor by $\gamma_{5}$. Since this corresponds to a change of basis, this choice of sign for the mass term does not affect the results for the inner products.
} 
Specifically, the radial integral must converge at its lower limit. It contains a factor of $r$, i.e. $\int_{0} d r r$, whereas the Bessel functions behave, for $k r \ll 1$, as $J_{\nu} \propto r^{\nu}$ (see (22)).

When $n_{1,2}+\epsilon \in(-1,0)$, we have chosen $\sigma_{1,2}=-1$ so that the solutions $\chi_{k}$ remain square normalizable for all values of magnetic flux $\Phi$. Even though both signs are allowed by square normalizability for $\Phi \neq 0$, our choice of sign gives solutions that, in the zero flux limit, join smoothly onto the cylindrical wave solutions to the noninteracting massive Dirac equation $(i \not \partial+m) \psi=0$.

It is worth expanding upon this ambiguity and the resolution we have adopted here. This is a subtle issue discussed first in context of strings in Ref. [6] (see also [7], [8] and [9]). The basic problem is that for certain angular momentum channels there are four solutions to the radial equation that are normalizable rather than two (for a given energy and z-momentum). Thus additional boundary conditions must be specified at the origin to determine the two physical solutions. The set of possible boundary conditions is restricted by the condition that only those solutions are permissible that have zero radial current at the origin ("self-adjoint boundary conditions"). However this does not by itself fully specify the boundary conditions and some additional physical principle or regulation scheme must be invoked. In effect we have made a special choice of boundary conditions above. Our choice is natural and well-motivated for the following reasons: (1) It extrapolates smoothly to the case of integer flux. (2) The transition rate calculated using this boundary condition agrees with the perturbative result in their common domain of validity (see Sec. VF below). (3) One can imagine natural regulation schemes in which this boundary condition will arise [7]. Still it must be kept in mind that the results would come out different if different boundary conditions were used.

Shifting $n_{1,2}$ in (52) by an integer, $n_{1,2} \rightarrow n_{1,2}+m$ with $m \in \mathbb{Z}$, takes us from one solution to another. Hence we can absorb the integer part of $\epsilon$ in the label $n_{1,2}$ and, without loss of generality, choose the AB phase $\epsilon$ to lie in the interval $[0,1)$. We will denote the fractional part of $\epsilon$ as $\kappa$, defined by

$$
\kappa=\epsilon \bmod 1
$$

In terms of $\kappa$, note that the set of mode functions here (and the set with $k_{z} \neq 0$ below) is now manifestly periodic in the $\mathrm{AB}$ phase $e \Phi$. Hence the radiation rate that follow will also enjoy this periodicity.

\section{Modes with arbitrary $k_{z}$}

Above we have found all modes with $k_{z}=0$. Now we perform boosts along the solenoid to obtain modes with $k_{z} \neq 0$. Denote the Lorentz boost by $\mathcal{B}$ and so

$$
\chi_{k} \rightarrow \mathcal{B} \cdot \chi_{k}
$$

Under the transformation

$$
t \rightarrow t \cosh \eta-z \sinh \eta
$$

with the rapidity parameter $\eta$ defined through

$$
\tanh \eta=\frac{k_{z}}{k^{0}}
$$

the boost matrix $\mathcal{B}$ is

$$
\begin{aligned}
\mathcal{B} & =U \cdot \mathcal{B}_{c} \cdot U^{-1}, \\
\mathcal{B}_{c} & \equiv\left[\begin{array}{cc}
e^{\eta / 2} \frac{1-\sigma^{3}}{2}+e^{-\eta / 2} \frac{1+\sigma^{3}}{2} & 0 \\
0 & e^{\eta / 2} \frac{1+\sigma^{3}}{2}+e^{-\eta / 2} \frac{1-\sigma^{3}}{2}
\end{array}\right]
\end{aligned}
$$

Denote $\chi_{\mathrm{I}}$ as the boosted $\chi_{k}$ with $\mathcal{N}_{\mathrm{B}}=0$; and $\chi_{\mathrm{II}}$ as the boosted $\chi_{k}$ with $\mathcal{N}_{\mathrm{A}}=0$. The two independent solutions for each set of positive and negative energy wavefunctions, in the Lorenz gauge for $A_{\mu}$ (49), are then

$$
\begin{aligned}
& \chi_{\left(\mathrm{I}, n, k, k_{z} \mid \pm\right)}(t, r, \theta, z) \\
& =\left[\begin{array}{c}
\cosh (\eta / 2) J_{\sigma(\kappa+n+1)}(k r) e^{i \theta} \\
\cosh (\eta / 2) \sigma \lambda_{ \pm}(k) J_{\sigma(\kappa+n)}(k r) \\
-i \sinh (\eta / 2) J_{\sigma(\kappa+n+1)}(k r) e^{i \theta} \\
i \sinh (\eta / 2) \sigma \lambda_{ \pm}(k) J_{\sigma(\kappa+n)}(k r)
\end{array}\right] \\
& \times \mathcal{N}_{ \pm}\left(k, k_{z}\right) e^{\mp i k_{0} t} e^{i n \theta} e^{ \pm i k_{z} z}, \\
& \chi_{\left(\mathrm{II}, n^{\prime}, k^{\prime}, k_{z}^{\prime} \mid \pm\right)}(t, r, \theta, z) \\
& {\left[\begin{array}{c}
i \sinh \left(\eta^{\prime} / 2\right) \sigma^{\prime} \lambda_{ \pm}\left(k^{\prime}\right) J_{\sigma^{\prime}\left(\kappa+n^{\prime}+1\right)}\left(k^{\prime} r\right) e^{i \theta} \\
-i \sinh \left(\eta^{\prime} / 2\right) J_{\sigma^{\prime}\left(\kappa+n^{\prime}\right)}\left(k^{\prime} r\right) \\
\cosh \left(\eta^{\prime} / 2\right) \sigma^{\prime} \lambda_{ \pm}\left(k^{\prime}\right) J_{\sigma^{\prime}\left(\kappa+n^{\prime}+1\right)}\left(k^{\prime} r\right) e^{i \theta} \\
\cosh \left(\eta^{\prime} / 2\right) J_{\sigma^{\prime}\left(\kappa+n^{\prime}\right)}\left(k^{\prime} r\right) \\
\times \mathcal{N}_{ \pm}\left(k^{\prime}, k_{z}^{\prime}\right) e^{\mp i k^{\prime}{ }_{0} t} e^{i n^{\prime} \theta} e^{ \pm i k_{z}^{\prime} z},
\end{array}\right]}
\end{aligned}
$$

now with

$$
\sigma, \sigma^{\prime}= \begin{cases}1 & \text { if } n, n^{\prime} \geq 0 \\ -1 & \text { if } n, n^{\prime} \leq-1\end{cases}
$$

and

$$
\begin{gathered}
\lambda_{ \pm}(k) \equiv \frac{k}{m+\omega_{ \pm}}, \quad \mathcal{N}_{ \pm}\left(k, k_{z}\right) \equiv \sqrt{\frac{\omega_{ \pm}+m}{2 k_{0}}} \\
k, k^{\prime}>0 ; \quad k_{z}, k_{z}^{\prime} \in \mathbb{R} ; \quad n, n^{\prime}=0, \pm 1, \pm 2, \ldots
\end{gathered}
$$

We have normalized our solutions using

$$
\begin{aligned}
\int_{0}^{\infty} d r \quad r J_{\nu}(k r) J_{\nu}\left(k^{\prime} r\right) & =\frac{\delta\left(k-k^{\prime}\right)}{\sqrt{k k^{\prime}}}, \\
k, k^{\prime}>0, \operatorname{Re}(\nu)>-1 & \\
\int_{0}^{2 \pi} d \theta e^{i\left(n-n^{\prime}\right) \theta} & =2 \pi \delta_{n, n^{\prime}}, \quad n, n^{\prime} \in \mathbb{Z} \\
\int_{-\infty}^{+\infty} d z e^{i\left(q-q^{\prime}\right) z} & =2 \pi \delta\left(q-q^{\prime}\right), \quad q, q^{\prime} \in \mathbb{R}
\end{aligned}
$$


such that

$$
\begin{aligned}
& \int d^{3} x^{\prime} \chi_{\left(A, n, k, k_{z} \mid \sigma_{E}\right)}^{\dagger}\left(\mathbf{x}^{\prime}\right) \chi_{\left(A^{\prime}, n^{\prime}, k^{\prime}, k_{z}^{\prime} \mid \sigma_{E}^{\prime}\right)}\left(\mathbf{x}^{\prime}\right) \\
& =\delta_{\sigma_{E}, \sigma_{E}^{\prime}} \delta_{A, A^{\prime}} \delta_{n, n^{\prime}}(2 \pi)^{2} \frac{\delta\left(k-k^{\prime}\right) \delta\left(k_{z}-k_{z}^{\prime}\right)}{\sqrt{k k^{\prime}}} \\
& \sigma_{E}, \sigma_{E}^{\prime}= \pm, A, A^{\prime} \in\{\mathrm{I}, \mathrm{II}\}
\end{aligned}
$$

We will be comparing the results here to those in Sec. III, where perturbation theory was performed with plane wave solutions written in Cartesian coordinates. To make the comparison, it is worthwhile to note that the normalization in (57) is consistent with the $\left\langle\mathbf{k} \mid \mathbf{k}^{\prime}\right\rangle=$ $(2 \pi)^{3} \delta^{3}\left(\mathbf{k}-\mathbf{k}^{\prime}\right)$ one would otherwise have obtained if Cartesian coordinates were utilized. Moreover,

$$
\begin{aligned}
& (2 \pi)^{3} \delta^{3}\left(\mathbf{k}-\mathbf{k}^{\prime}\right) \\
& \quad=(2 \pi)^{3} \delta\left(\phi-\phi^{\prime}\right) \delta\left(k-k^{\prime}\right) \delta\left(k_{z}-k_{z}^{\prime}\right)\left(k k^{\prime}\right)^{-1 / 2} .
\end{aligned}
$$

The $\sqrt{k k^{\prime}}$ in (57) is the (symmetrized) Jacobian when transforming from Cartesian to cylindrical coordinates. The $(2 \pi) \delta\left(\phi-\phi^{\prime}\right)$ is the completeness relation

$$
2 \pi \delta\left(\phi-\phi^{\prime}\right)=\sum_{n=-\infty}^{+\infty} e^{i n\left(\phi-\phi^{\prime}\right)}, \quad\left|\phi-\phi^{\prime}\right| \in[0,2 \pi),
$$

Denoting our mode functions here by $\left|n, k, k_{z}\right\rangle$ (and suppressing the $A$ and $\sigma_{E}$ dependence), we see that (58)

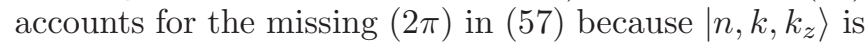
the $n$th term in the Fourier series expansion of $|\mathbf{k}\rangle$, the mode functions written in Cartesian coordinates, since

$$
|\mathbf{k}\rangle=\left|k, k_{z}, \phi\right\rangle=\sum_{n}\left|k, k_{z}, n\right\rangle e^{i n \phi} .
$$

and hence (57) yields

$$
\sum_{n, n^{\prime}}\left\langle k, k_{z}, n \mid k^{\prime}, k_{z}^{\prime}, n^{\prime}\right\rangle e^{i n^{\prime} \phi^{\prime}} e^{-i n \phi}=\left\langle\mathbf{k} \mid \mathbf{k}^{\prime}\right\rangle .
$$

\section{Transformation to Axial Gauge}

The above modes for a stationary solenoid, $\xi=0$, have been found in Lorenz gauge. However, to find overlaps and the radiation rate, it is easier to work in axial gauge. Hence we now transform the modes to axial gauge with $A_{\mu}$ in (48),

$$
A_{\mu}=(0,0,-\Phi \Theta(x) \delta(y), 0), \quad \text { (Cartesian) }
$$

The $\chi$ solutions differ from its Lorenz gauge counterpart in (55) only by a phase factor

$$
\chi_{(\text {axial })}=e^{i \kappa(\theta \bmod 2 \pi)} \chi_{(\text {Lorenz, Eq [55) }}
$$

In (59), to see it is $\kappa$ and not $\epsilon$ that should occur in the phase, refer to the solutions (52) before the introduction of $\kappa$, and note that the axial gauge version of (52) would contain a $e^{i \epsilon(\theta \bmod 2 \pi)}$. The $e^{i \kappa(\theta \bmod 2 \pi)}$ in (59) would then follow from the re-definition of the Fourier mode label $n$ that introduced $\kappa$.

\section{E. Matrix Elements for Moving Solenoid}

With the solutions (59) in hand, we are now ready to evaluate the integral (43). It is only necessary to calculate (43) for positive and negative solutions from (59) with Fourier mode labels $\left(n, n^{\prime}\right)=(-1,0)$ and $(0,-1)$, where the $n$ refers to the positive energy solution and $n^{\prime}$ to the negative energy solution. For other values of $n$ and $n^{\prime}$, the factor of $\partial_{x} \chi_{\left(s^{\prime} \mid-\right)}$ in Eq. (43) yields the difference of two negative energy solutions in (59), which by (57) has zero overlap with all its positive energy counterparts.

For $n^{\prime}=0, \partial_{x} \chi_{\left(s^{\prime} \mid-\right)}^{\left(\sigma^{\prime}=+1\right)}$ is a linear combination of the $n^{\prime}=-1$ and the $n^{\prime}=+1$ negative energy solutions in (59), except the former has $\sigma^{\prime}=+1$. For $n^{\prime}=-1$, $\partial_{x} \chi_{\left(s^{\prime} \mid-\right)}^{\left(\sigma^{\prime}=-1\right)}$ is a linear combination of the $n^{\prime}=-2$ and $n^{\prime}=0$ negative energy solutions in (59), with the latter's $\sigma^{\prime}=-1$. In addition, the $n^{\prime}=-1$ solution with $\sigma^{\prime}=+1$ in $\partial_{x} \chi_{\left(n^{\prime}=0 \mid-\right)}$ remains orthogonal to all positive energy solutions except possibly for the $n=-1$ case; likewise the $n^{\prime}=0$ solution with $\sigma^{\prime}=-1$ in $\partial_{x} \chi_{\left(n^{\prime}=-1 \mid-\right)}$ is orthogonal to all $\chi_{(s \mid+)}$ except perhaps $\chi_{(n=0 \mid+)}$.

As we will see in a moment, these two overlap integrals,

$$
\int d^{3} x^{\prime}\left[\chi_{(n=-1 \mid+)}^{(\sigma=-1)}\right]^{\dagger} \chi_{\left(n^{\prime}=-1 \mid-\right)}^{\left(\sigma^{\prime}=+1\right)}
$$

and

$$
\int d^{3} x^{\prime}\left[\chi_{(n=0 \mid+)}^{(\sigma=+1)}\right]^{\dagger} \chi_{\left(n^{\prime}=0 \mid-\right)}^{\left(\sigma^{\prime}=-1\right)}
$$

do yield non-zero answers and are therefore the only ones contributing to the pair production rate.

Computing the volume integral in (43) for solutions with the Fourier modes given by $\left(n, n^{\prime}\right)=(-1,0)$ and $(0,-1)$ requires the integral

$$
\begin{aligned}
& \int_{0}^{\infty} d r r J_{\nu}(q r) J_{-\nu}(k r) \\
&= \operatorname{Pr}\left(\frac{2 \sin (\pi \nu)}{\pi\left(q^{2}-k^{2}\right)}\left(\frac{q}{k}\right)^{\nu}\right)+\frac{\cos (\pi \nu)}{\sqrt{q k}} \delta(k-q), \\
& k, q>0, \operatorname{Re}[\nu]>-1
\end{aligned}
$$

where Pr denotes the principal value.

Define

$\mu_{++} \equiv \sqrt{\frac{1}{\omega_{k}}+\frac{1}{k_{0}}} \sqrt{\frac{1}{\omega_{k^{\prime}}}+\frac{1}{k_{0}^{\prime}}}-\sqrt{\frac{1}{\omega_{k}}-\frac{1}{k_{0}}} \sqrt{\frac{1}{\omega_{k^{\prime}}}-\frac{1}{k_{0}^{\prime}}}$

and

$\mu_{+-} \equiv \sqrt{\frac{1}{\omega_{k}}+\frac{1}{k_{0}}} \sqrt{\frac{1}{\omega_{k^{\prime}}}-\frac{1}{k_{0}^{\prime}}}-\sqrt{\frac{1}{\omega_{k}}-\frac{1}{k_{0}}} \sqrt{\frac{1}{\omega_{k^{\prime}}}+\frac{1}{k_{0}^{\prime}}}$.

The result then reads 


$$
\begin{gathered}
\left(\int d^{3} x^{\prime} \chi_{\left(A, n, k, k_{z} \mid+\right)}^{\dagger}\left(\mathbf{x}^{\prime}\right) \partial_{x} \chi_{\left(A^{\prime}, n^{\prime}, k^{\prime}, k_{z}^{\prime} \mid-\right)}\left(\mathbf{x}^{\prime}\right)\right)_{k \neq k^{\prime}}=-\frac{1}{2} \delta\left(k_{z}+k_{z}^{\prime}\right) \sin (\pi \kappa) \sqrt{k k^{\prime}} \\
\times\left[\delta _ { n , 0 } \delta _ { n ^ { \prime } , - 1 } ( \frac { k } { k ^ { \prime } } ) ^ { \kappa } \left\{\frac{\mu_{++}}{\omega_{k}+\omega_{k^{\prime}}}\left(\delta_{A}^{\mathrm{I}} \delta_{A^{\prime}}^{\mathrm{I}} k \sqrt{\frac{\omega_{k^{\prime}}-m}{\omega_{k}+m}}+\delta_{A}^{\mathrm{II}} \delta_{A^{\prime}}^{\mathrm{II}} k^{\prime} \sqrt{\frac{\omega_{k}+m}{\omega_{k^{\prime}}-m}}\right)\right.\right. \\
\left.+\frac{\mu_{+-} \operatorname{sgn}\left(k_{z}^{\prime}\right)}{\left(\omega_{k}-\omega_{k^{\prime}}\right) \sqrt{\omega_{k}+m} \sqrt{\omega_{k^{\prime}}-m}}\left(\delta_{A}^{\mathrm{I}} \delta_{A^{\prime}}^{\mathrm{II}} k k^{\prime}-\delta_{A}^{\mathrm{II}} \delta_{A^{\prime}}^{\mathrm{I}}\left(\omega_{k}+m\right)\left(\omega_{k^{\prime}}-m\right)\right)\right\} \\
-\delta_{n,-1} \delta_{n^{\prime}, 0}\left(\frac{k^{\prime}}{k}\right)^{\kappa}\left\{\frac{\mu_{++}}{\omega_{k}+\omega_{k^{\prime}}}\left(\delta_{A}^{\mathrm{I}} \delta_{A^{\prime}}^{\mathrm{I}} k^{\prime} \sqrt{\frac{\omega_{k}+m}{\omega_{k^{\prime}}-m}}+\delta_{A}^{\mathrm{II}} \delta_{A^{\prime}}^{\mathrm{II}} k \sqrt{\frac{\omega_{k^{\prime}}-m}{\omega_{k}+m}}\right)\right. \\
\left.\left.+\frac{\mu_{+-i \operatorname{sgn}\left(k_{z}^{\prime}\right)}}{\left(\omega_{k}-\omega_{k^{\prime}}\right) \sqrt{\omega_{k}+m} \sqrt{\omega_{k^{\prime}}-m}}\left(\delta_{A}^{\mathrm{I}} \delta_{A^{\prime}}^{\mathrm{II}}\left(\omega_{k}+m\right)\left(\omega_{k^{\prime}}-m\right)-\delta_{A}^{\mathrm{II}} \delta_{A^{\prime}}^{\mathrm{I}} k k^{\prime}\right)\right\}\right]
\end{gathered}
$$

Direct substitution of the Bessel integral Eq. 600 would lead to an additional term in the transition amplitude above that is proportional to $\delta\left(k-k^{\prime}\right)$. We have omitted this term following the discussion in Ref. [3]. As explained there, the integrals must be carefully regulated in order to exclude spurious terms that lead to particle production even in the limit of zero flux. This is accomplished by performing the integrals over a finite volume, imposing suitable boundary conditions, and then taking the infinite volume limit. Such an analysis eliminates the term proportional to $\delta\left(k-k^{\prime}\right.$ ) (see Sec. IIIB of ref [3] for more details).

The expression in (61) yields 8 different channels for pair production to occur. Moreover, at the level of individual amplitudes, the periodic dependence on the magnetic flux is already manifest, since they depend on the $\mathrm{AB}$ phase only via $\kappa=\epsilon \bmod 1$.

\section{F. Moving frames pair production rate}

Inserting (61) into (43) and summing the squares of the resulting individual amplitudes then gives us the rate of fermion-antifermion pair production per unit length of the infinite solenoid

$$
\begin{aligned}
\dot{N}^{\prime}=\int_{0}^{\infty} & d k k \int_{0}^{\infty} d k^{\prime} k^{\prime} \int_{-\infty}^{\infty} d k_{z} \int_{-\infty}^{\infty} d k_{z}^{\prime} \\
& \times \delta\left(k_{0}+k_{0}^{\prime}-\Omega\right) \delta\left(k_{z}+k_{z}^{\prime}\right) \\
& \times \frac{v_{0}^{2} \sin ^{2}(\pi \kappa)}{8 \pi^{2} \Omega^{2} k_{0} k_{0}^{\prime}}\left(m^{2}+k_{z}^{2}+k_{0} k_{0}^{\prime}\right) \\
& \times\left[\left(\frac{k}{k^{\prime}}\right)^{2 \kappa}+\left(\frac{k^{\prime}}{k}\right)^{2 \kappa}\right]
\end{aligned}
$$

In the limit when $\kappa \ll 1$, the last term (in parenthesis) goes to 2 and $\sin ^{2}(\pi \kappa) \approx \pi^{2} \kappa^{2}$, and

$$
\dot{N}^{\prime}=\frac{v_{0}^{2} \kappa^{2}}{4 \Omega^{2}} \int_{0}^{\infty} d k k \int_{0}^{\infty} d k^{\prime} k^{\prime} \int_{-\infty}^{\infty} d k_{z} \int_{-\infty}^{\infty} d k_{z}^{\prime}
$$

$$
\times \delta\left(k_{0}+k_{0}^{\prime}-\Omega\right) \delta\left(k_{z}+k_{z}^{\prime}\right)\left(1+\frac{k_{z}^{2}+m^{2}}{k_{0} k_{0}^{\prime}}\right)
$$

This agrees with (23), the non-relativistic limit of the interaction picture perturbation theory result, if we identify $\kappa \leftrightarrow \epsilon$.

\section{CONCLUSIONS}

We have solved for fermionic radiation first from oscillating electromagnetic solenoids and then from cosmic string loops. For the solenoid we have done the calculation in two different ways, first using a small $\mathrm{AB}$ phase approximation, and second by considering slowly moving solenoids. We have evaluated the angular distribution of the fermionic radiation from the solenoid, and the total power emitted from cosmic string loops and cusps. Our results can be compared to the results of Ref. [3].

The total power emitted in bosons and fermions is very comparable. For example, both are proportional to $\epsilon^{2} v_{0}^{2} \Omega^{2}$ for the lowest harmonic of the oscillating solenoid (see Eq. (24)). However, the angular distributions of the radiation in the two cases are quite distinct. To highlight the difference, we show the angular distribution in both cases for the lowest harmonic emission from an oscillating solenoid in Fig. 2. We also find that the fermion and antifermion are preferably emitted in opposite helicity states and discuss the spin distribution. Just like in the bosonic case, fermionic $\mathrm{AB}$ radiation from kinks and cusps on cosmic strings is ultra-violet divergent for massless fermions, with a linear dependence on the cut-off. This may translate into a significant amount of radiation of neutrinos from strings with which neutrinos have an $\mathrm{AB}$ interaction.

Our results ought to apply to the low energy end of the emission spectrum of electrons from idealized solenoids, where the wavelengths of the particle pairs are much longer than the diameter of the solenoid. A more realistic theoretical investigation would have to take into account the finite width of the solenoid itself. 
In Ref. [3], the gravitational analog of $\mathrm{AB}$ radiation was also discussed. Via the same analogy, we also expect cosmic strings to radiate fermions. We leave that calculation for future work.

\section{Acknowledgments}

We thank Roman Jackiw for comments. This work was supported by the U.S. Department of Energy at Case
Western Reserve University. TV was also supported by grant number DE-FG02-90ER40542 at the Institute for Advanced Study. Much of the numerical and analytic work in this paper was done with Mathematica [10].
[1] Y. Aharonov and D. Bohm, Phys. Rev. 115, 485 (1959).

[2] M. G. Alford and F. Wilczek, Phys. Rev. Lett. 62, 1071 (1989).

[3] K. Jones-Smith, H. Mathur and T. Vachaspati, Phys. Rev. D81, 043503 (2010); arXiv:0911.0682 [hep-th].

[4] J. Garriga, D. Harari and E. Verdaguer, Nucl. Phys. B 339, 560 (1990).

[5] P.M. Morse and H. Feshbach, "Methods of Theoretical Physics," Volume I, McGraw-Hill (1953)

G.N. Watson, "A Treatise On The Theory Of Bessel Functions," 2nd Ed., Cambridge (1944)

[6] Ph. de Sousa Gerbert and R. Jackiw, Commun. Math. Phys. 124, 229 (1989).

[7] M.G. Alford, J. March-Russell and F. Wilczek, Nucl Phys B328, 140 (1989).

[8] R. Jackiw, A.I. Milstein, S.-Y. Pi, and I.S. Terekhov, Phys Rev B80, 033413 (2009).

[9] A. Roy and M. Stone, J. Phys. A43, 015203 (2010).

[10] Wolfram Research, Inc., Mathematica, Champaign, IL (2005)

\section{Appendix A: Conventions}

Our metric and spacetime index convention are defined by $\eta_{\mu \nu}=\operatorname{diag}(1,-1,-1,-1), x^{\mu}=(t, x, y, z)$ with $\mu=0,1,2,3$. Whenever $x$ appears alone, without indices attached, it always means the $\mu=1$ component of $x^{\mu}$. The Einstein summation convention always applies unless otherwise stated. The spacetime inner product of $a^{\mu}$ and $b^{\mu}$ is $a \cdot b$; while $a^{2} \equiv a_{\mu} a^{\mu}$. Bold fonts denote spatial vectors; for instance, $\mathbf{a} \cdot \mathbf{b}=\delta_{i j} a^{i} b^{j}$ and $\mathbf{a}^{2}=\delta_{i j} a^{i} a^{j}$.

Dirac action: The action in (1) define the dynamics of a Dirac fermion interacting with the photon vector potential $A_{\mu}$. We make use of the Feynman slash notation. In particular,

$$
\not D \psi=\gamma^{\mu}\left(\partial_{\mu}+i e A_{\mu}\right) \psi
$$

Except in section (V), we employ the chiral basis for the $\left\{\gamma^{\mu}\right\}$ matrices,

$$
\begin{aligned}
& \gamma^{\mu}=\left[\begin{array}{cc}
0 & \sigma^{\mu} \\
\bar{\sigma}^{\mu} & 0
\end{array}\right], \\
& \sigma^{\mu}=\left(\mathbf{1}_{2 \times 2}, \sigma^{k}\right), \quad \bar{\sigma}^{\mu}=\left(\mathbf{1}_{2 \times 2},-\sigma^{k}\right)
\end{aligned}
$$

which is, in turn, defined via the Pauli matrices,

$$
\sigma^{1} \equiv\left[\begin{array}{ll}
0 & 1 \\
1 & 0
\end{array}\right], \quad \sigma^{2} \equiv\left[\begin{array}{cc}
0 & -i \\
i & 0
\end{array}\right], \quad \sigma^{3} \equiv\left[\begin{array}{cc}
1 & 0 \\
0 & -1
\end{array}\right]
$$

Spinorial solutions: Except in section V interaction picture perturbation theory will be used. It is carried out using the plane wave solutions to the non-interacting massive Dirac equation $(i \not \partial-m) \psi=0$, where the positive energy $\psi_{(k, s \mid+)}$ and negative energy $\psi_{(k, s \mid-)}$ solutions with momentum $k$ and spin $s$ are

$$
\begin{aligned}
& \psi_{(k, s \mid+)} \equiv \frac{e^{-i k \cdot x}}{\sqrt{2 k_{0}}} u_{k}^{s} \equiv \frac{e^{-i k \cdot x}}{\sqrt{2 k_{0}}}\left[\begin{array}{c}
\sqrt{\sigma \cdot k} \xi^{s} \\
\sqrt{\bar{\sigma} \cdot k} \xi^{s}
\end{array}\right], \\
& \psi_{(k, s \mid-)} \equiv \frac{e^{+i k \cdot x}}{\sqrt{2 k_{0}}} v_{k}^{s} \equiv \frac{e^{+i k \cdot x}}{\sqrt{2 k_{0}}}\left[\begin{array}{c}
\sqrt{\sigma \cdot k} \xi^{s} \\
-\sqrt{\bar{\sigma} \cdot k} \xi^{s}
\end{array}\right]
\end{aligned}
$$

where

$$
\left(\xi^{s}\right)_{a}=\delta_{a}^{s}, \quad k_{0}=\sqrt{\mathbf{k}^{2}+m^{2}} .
$$

The $\sqrt{\sigma \cdot k}$ and $\sqrt{\bar{\sigma} \cdot k}$ are the matrices $\sigma \cdot p$ and $\bar{\sigma} \cdot p$ written in diagonalized form (i.e. $U D U^{-1}$, where $D$ is diagonal), with the eigenvalues replaced with their positive square roots. By going to the rest frame of the particle, it can be seen that the $\xi^{1}$ and $\xi^{2}$ are the spin up $(s=1)$ and spin down $(s=2)$ states for the fermion with respect to the basis of Pauli matrices used here. For the antifermion, they are the spin down $\left(s^{\prime}=1\right)$ and spin up $\left(s^{\prime}=2\right)$ states. These plane wave solutions are normalized such that

$$
\int \psi_{(k, s \mid \pm)}^{\dagger} \psi_{\left(k^{\prime}, s^{\prime} \mid \pm\right)} d^{3} x=(2 \pi)^{3} \delta^{(3)}\left(\mathbf{k}-\mathbf{k}^{\prime}\right) \delta_{s^{\prime}}^{s}
$$

States of definite helicity are obtained by taking the spinors $\xi$ to be eigenspinors of $\boldsymbol{\sigma} \cdot \mathbf{k}$ with eigenvalues $\pm|\mathbf{k}|$. For the particle the positive eigenvalue corresponds to positive helicity and negative to negative; for the antiparticle the negative eigenvalue corresponds to positive helicity and vice-versa. 